\title{
Experimental validation of a dimensional analysis of spheronisation of cylindrical extrudates
}

\author{
J. Parkin, K.S. Widjaja, M.P. Bryan, S.L. Rough* and D.I. Wilson \\ University of Cambridge, Department of Chemical Engineering and Biotechnology, \\ New Museums Site, Pembroke Street, Cambridge, CB2 3RA, UK
}

Submitted to

Powder Technology

(C) JP, KSW, MPB, SLR \& DiW

May 2016

*Corresponding author

Dr Sarah L. Rough

Department of Chemical Engineering and Biotechnology

New Museums Site

Pembroke Street

Cambridge

CB2 3RA, UK

E-mail slr1002@cam.ac.uk

Tel. $\quad+441223334777$

Fax $\quad+441223334796$ 


\title{
A dimensional analysis of spheronisation of cylindrical extrudates
}

\author{
J. Parkin, K.S. Widjaja, M.P. Bryan, S.L. Rough* and D.I. Wilson \\ University of Cambridge, Department of Chemical Engineering and Biotechnology, \\ New Museums Site, Pembroke Street, Cambridge, CB2 3RA, UK
}

\begin{abstract}
Extrusion-spheronisation (E-S) is a widely used technique for the manufacture of pellets with high sphericity and narrow size distribution. A dimensionless framework for describing the evolution of pellet shape with spheronisation time is presented.

The experiments employed a $45 \mathrm{wt} \%$ microcrystalline cellulose (MCC)/water-based paste, with up to $15 \mathrm{wt} \%$ calcium carbonate added to represent a 'hard' active pharmaceutical ingredient (API). The bulk yield strength of the paste was measured and found to increase with increasing carbonate content, which in turn led to higher extrusion pressures, longer spheronisation times and less spherical pellets for a given set of operating parameters. The pellet aspect ratio was found to increase in a linear manner with the logarithm of spheronisation time, progressing towards an asymptotic final value. This behaviour is compared with two simple models.
\end{abstract}

The dimensional analysis of the pellet rounding stage identified the paste density and bulk yield strength as scaling quantities. The model borne from the dimensional analysis was validated and was found to be applicable to a second material (MCC/lactose/water).

High speed imaging was also used to examine the collision behaviour of pellets during the breakage and rounding stages in spheronisation. The results confirmed that the rounding phase was the rate-determining step. The velocities of a number of tracked pellets were also quantified, and were consistently lower than the tip speed of the rotating friction plate.

Keywords: dimensional analysis, extrusion-spheronisation, microcrystalline cellulose, pellet shape, rounding 


\section{Introduction}

Extrusion-spheronisation (E-S) is used in the pharmaceutical and agrichemical sectors for manufacturing pellets with high sphericity and density [2]. E-S is a two stage granulation process in which the particulate solids are firstly combined with a liquid (the binder) to give a wet mass (also termed 'dough' or 'paste') that is then extruded through dies or screens to give cylindrical extrudates of random length but uniform diameter. The extrudates are subsequently spheronised on a rotating friction plate to give pellets $[3,4]$. The term 'pellet' is used here to label the assembly of primary particles, which may be a mixture of different constituents. Spheronisation differs from pan granulation (where initially free flowing particles are combined with liquid on a rotating dish) in that the starting materials are a wet mass rather than a powder.

The mechanism of spheronisation involves a number of stages and three qualitative models have been presented in the E-S literature. In the model of Rowe [5], cylindrical extrudates break into short lengths as a result of collisions with each other, the friction plate and the walls of the spheroniser. The rods undergo plastic deformation and become rounded cylinders: these are further rounded to a dumb-bell, then to an ellipsoid or egg-shape and finally to a sphere. Baert et al. [6] reported that twisting of the extrudates played an important part in their breakage and subsequent rounding. Liew et al. [7] stated that fines generated at the point of extrudate breakage and by attrition during collisions played an important role in rounding, which was supported by the experimental studies of Koester et al. [8]. The tendency to generate fines, which favours the Liew et al. mechanism over that of Rowe, was shown by Bryan et al. [9] to be related to the processing history of the paste. Bryan et al. studied the effect of mixing on notionally identical formulations of microcrystalline cellulose/water pastes and found that the use of a mixer imposing a larger shear strain rate resulted in differences in the paste rheology as well as the subsequent spheronisation behaviour. In particular, noticeably larger amounts of fines were generated when extrudates prepared by high shear mixing of the paste were spheronised.

Knowledge of the phenomenology of spheronisation is important as this can guide simulations of pellet breakage and plastic deformation in constructing quantitative physical models. Detailed simulations (e.g. see Sinka [10, 11]) require reliable estimates of the physical properties of the pellets (and their interaction with the components of the spheronisation device), their interdependency and thus evolution over the course of the 
process. Simulations employing the discrete element method (DEM), such as those reported by Sinka and co-workers $[11,12]$, are currently being developed for E-S but are not yet able to assist the interpretation of experimental data or design of formulations. Detailed simulations also require data for verification of predictions such as pellet motion and evolution in microstructure. In this regard, the distribution of instantaneous pellet positions and velocities is becoming better understood as a result of developments in measurement techniques (e.g. [13], [14]). Instantaneous measurements of pellet shape, porosity and composition distribution are, by comparison, scarce. In the period until detailed simulations are able to provide a priori estimates of E-S mechanisms and the influence of operating parameters, it is appropriate to employ classical modelling techniques such as those based on dimensional analysis to develop semi-empirical descriptions for processes as complex as E-S. This paper presents the development of such a model to describe the evolution of pellet size and shape for extrudates which undergo spheronisation by the Rowe mechanism, i.e. without the influence of fines.

Data describing the evolution of pellet number, size and shape were collected from experiments using the approach reported by Lau et al. [15] to investigate the spheronisation of a simple microcrystalline cellulose (MCC)/water paste. E-S formulations of water-tolerant active pharmaceutical ingredients (APIs) regularly employ MCC as an excipient, as this 'gold standard' material [14] provides the paste with good water retention properties and ductility. Lau et al. [15] performed spheronisation tests starting with a set number (20) of identical extrudates, stopping tests after different times in order to measure the number of pellets, their size and shape. With this relatively small number of extrudates, collisions were dominated by pellet-spheroniser wall events. This was confirmed in the current study by high speed video monitoring of the spheronisation step. Lau et al. did not observe twisting, associated with the Baert et al. [6] mechanism, whilst subsequent studies on similar MCC/water pastes by Bryan et al. [1] indicated that the mechanism of fines specifically collecting at the waist (as described by Liew et al. [7]) was not responsible for the transition from dumb-bell to more spherical shape.

Dimensional analysis has not, to the authors' knowledge, been applied to the dynamics of spheronisation. Our analysis yields relationships which were tested against two new sets of experimental data: one to identify the functional form, and a second to validate the trends therein. 
The dimensional analysis reported here assumes that the change in pellet shape during spheronisation arises from viscoplastic deformation (e.g. [11], [16]) characterised by a plastic bulk yield strength, $\sigma_{\mathrm{Y}}$. This yield strength is determined by paste formulation and mixing history (see [9]), and was investigated here using a series of paste compositions. $\sigma_{\mathrm{Y}}$ was quantified experimentally by the Benbow-Bridgwater characterisation technique [17]. The $45 \mathrm{wt} \% \mathrm{MCC} /$ water paste studied by Bryan et al. [1] was used as a base matrix, as well as mixtures with 5, 10 and $15 \mathrm{wt} \%$ (wet basis) calcium carbonate (calcite polymorph) representing different levels of insoluble API. A second base formulation, with composition by mass 25:25:50 MCC, $\alpha$-lactose monohydrate and water, was also tested. This formulation was developed by scrutinising previous work on E-S ([18], [19], [20]). Dimensional analysis has not, to the authors' knowledge, been applied to the dynamics of spheronisation. Our analysis yields relationships which were tested against two new sets of experimental data: one to identify the functional form, and a second to validate the trends therein.

\section{Modelling}

\subsection{Dimensional analysis}

The pellet size is characterised, at the simplest level, by its length $l$ and breadth $b$ : its shape can then be quantified in terms of the aspect ratio, $A R$, defined as $A R=b / l$. Other measures of pellet shape exist and could be used as desired (see [1]). The parameters which determine the evolution of pellet shape (and thus progression to spheronisation end-point) are postulated from observations and inspection of the literature to be:

\section{Operating parameters}

$N \quad$ number of pellets

D diameter of extrudate (this will set the initial pellet breadth)

$H \quad$ characteristic size of spheroniser friction plate grooves or elements

$R \quad$ radius of spheroniser friction plate

$\omega \quad$ spheroniser rotational speed

$g$ gravitational acceleration (body force in addition to centripetal acceleration)

$t$ time

\section{Material parameters}

$\rho \quad$ density of pellet material 


$$
\begin{array}{ll}
\mu_{\mathrm{S}} & \text { pellet-spheroniser surface friction coefficient } \\
\mu_{\mathrm{p}} & \text { pellet-pellet friction coefficient } \\
\sigma_{\mathrm{Y}} & \text { material bulk yield strength }
\end{array}
$$

These parameters feature three dimensions, giving nine independent dimensionless groups. The proposed functional relationship takes the form:

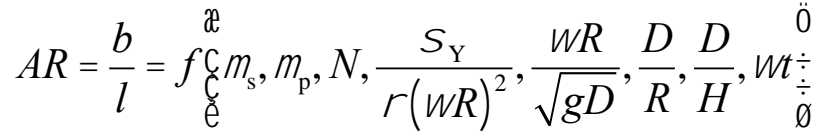

Table 1 summarises each group and the attribute it quantifies. The two friction coefficients are assumed to be constant as similar materials (MCC/water pastes) and the same spheroniser (with 316 stainless steel surfaces) were used in the tests. For the experiments reported here, the initial number of pellets, $N_{0}$, was fixed at 20 rods each with initial dimensions $D / l=3.5 / 21.5 \approx 1 / 6$. Each extrudate broke into three pellets, on average, in the initial stage of spheronisation. Breakage took approximately $1 / 10^{\text {th }}$ of the total spheronisation time. Equation (1) then simplifies to

$$
A R=\frac{b}{l}=f N_{0}, \frac{\mathrm{Y}}{(R)^{2}}, \frac{R}{\sqrt{g D}}, \frac{D}{R}, \frac{D}{H}, t \dot{\grave{!}}
$$

The effect of $\omega$ on the time to complete spheronisation, $t_{\text {end }}$, of MCC/water paste extrudates was investigated by Lau et al. [15]. They found that the time to complete spheronisation for a fixed initial number of extrudates scaled with spheroniser speed such that $\omega t_{\text {end }}{ }^{3.6}=$ constant. They presented a physically-based model, ignoring contributions from the Froude number, which gave $\omega t_{\text {end }}{ }^{3}=$ constant. Both expressions are consistent with Equation (2) when $A R$ is set at $A R_{\text {end }}$, some target for the process. Products of dimensionless groups giving a similar functional form to the latter expression include

$$
A R_{\text {end }}=f_{1} \frac{\mathrm{Y}}{(R)^{2}} \stackrel{1 / 2}{\dot{\vdots}} \quad \frac{R}{\sqrt{g D}} \dot{\doteqdot}\left(t_{\text {end }}\right)^{3} \dot{\div}
$$

or, with no contribution from the Froude number,

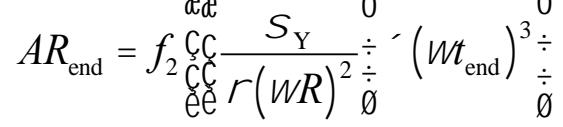

Further work is required to identify the form of the functional relationships. Increasing the initial load, i.e. increasing $N_{0}$, tends to extend $t_{\text {end }}(e . g$. [1]) and this result is consistent with 
Equation (2).

For the experiments conducted here, $N_{0}, D, H, R$ and $\omega$ were fixed, so Equation (2) condenses to

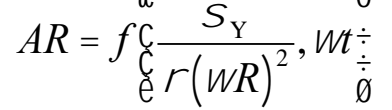

The bulk yield strength $\sigma_{\mathrm{Y}}$ is manipulated in these tests by changing the paste formulation. Fixing the spheroniser conditions $(H, R$ and $\omega)$ sets the maximum velocity that the pellet can attain (in effect, a multiple of the tip speed) and, to a first approximation, the pellet kinetic energy (densities are assumed not to vary strongly during spheronisation). This is likely to set the extent to which a pellet can be rounded, which is denoted as $A R_{\text {end }}$ :

$$
A R_{\text {end }}=f_{3}\left(\frac{\sigma_{\mathrm{Y}}}{\rho(\omega R)^{2}}\right) \quad t \rightarrow \infty
$$

It follows from the above that, in these tests, for a given set value of $A R_{\text {end }}, t_{\text {end }}$ will depend on $\sigma_{\mathrm{Y}}$ via

$$
\omega t_{\mathrm{end}}=g\left(\frac{\sigma_{\mathrm{Y}}}{\rho(\omega R)^{2}}\right)
$$

It follows that experimental data for a given paste formulation and spheroniser rotational speed should be compared using

$$
\frac{A R}{A R_{\text {end }}}=h\left(\frac{t}{t_{\text {end }}}\right)
$$

When the experimental data are plotted in the form suggested by Equation (8), it will be shown that they follow a dependency of the form $\left(A R / A R_{\text {end }}\right) \propto \log \left(t / t_{\text {end }}\right)$.

\subsection{Simple models}

The trends evident in the experimental data are compared with simple models for the evolution of pellet shape which give analytical results. It is anticipated that more detailed quantitative modelling approaches, including population balances, are needed to describe these systems.

For these simple models, we assume that the rate of collisions during spheronisation does not change over time, and that the result of a collision depends on how close the pellet is to being 
spherical. Let $y$ be a measure of pellet sphericity, defined as $0<y \leq 1$, where $y=1$ represents the final state. Plastic deformation caused by a collision is localised to the point of contact. As pellets become more spherical, only collisions where the point of contact lies on a protuberance, or where the pellet-wall or pellet-pellet collide directly (rather than obliquely), are postulated to result in a change (increase) in $y$.

\section{Pellet-wall collisions}

The wall does not change shape over time. A simple kinetic scheme is

$$
\mathrm{d} y=k(1-y) \mathrm{d} n
$$

where $n$ is the number of collisions and $k$ is a constant which is expected to depend on $\sigma_{\mathrm{Y}} / \rho(\omega R)^{2}$. Making the assumption that the rate of collision does not vary with time suggests that $\mathrm{d} n \propto \mathrm{d} t$, giving

$$
\mathrm{d} y=k_{1}(1-y) \mathrm{d} t
$$

with solution

$$
y=1-(1-y)_{0} \exp \left(-k_{1} t\right)
$$

Here, $(1-y)_{0}$ is the initial measure of approach to sphericity at the point from which rounding starts, which would correspond to the end of the breakage stage in these experiments.

\section{Pellet-pellet collisions}

Collision between two pellets would cause rounding of both, but it requires direct collision. The suggested kinetic scheme is

$$
2 \mathrm{~d} y=k(1-y)^{2} \mathrm{~d} n
$$

Applying the assumptions above gives

$$
\frac{1}{1-y}=\frac{1}{(1-y)_{0}}+k_{2} t
$$

Both Equations (11) and (13) yield loci which appear sigmoidal on a plot of $y$ against $\log _{10} t$ or $\ln t$, and have regions that appear linear.

In the experiments reported here, the aspect ratio is used as the measure of the approach to sphericity. This simple mapping is used for illustrative purposes as it allows the experimental data to be compared with the above results: the true relationship cannot be stated a priori without detailed phenomenological evidence. The data approach a final value, $A R_{\text {end }}$, which is near, but not equal to, 1. Equations (11) and (13) are then modified to fit the data by writing 
the above results as

$$
\begin{array}{ll}
\text { Model I } & A R=A R_{\text {end }}-\left(A R_{\text {end }}-A R\right)_{0} \exp \left(-k_{1} t\right) \\
\text { Model II } & \frac{1}{A R_{\text {end }}-A R}=\frac{1}{\left(A R_{\text {end }}-A R\right)_{0}}+k_{2} t
\end{array}
$$

These sigmoidal trends differ from those reported by Sinka [11], who modelled the effect of repeated impacts on a pellet initially of ellipsoidal shape, with deformation determined by a plasticity law. The point of impact was assigned randomly and all collisions were assumed to occur with the same velocity. Pellets with an initial aspect ratio of around 0.3 were predicted to become less spherical over time, whilst those with a value $>0.3$ were predicted to become more spherical over time, approaching $A R=1$ more rapidly with increasing time (and the number of collisions). This critical initial aspect ratio is expected to be determined by the model parameters. Sinka's rigorous treatment differs from the simple models above in the assumptions of constant collision velocity and direct impact, which are expected to be unrealistic and has promoted further work by that group on collisions [12].

\section{Materials and Methods}

\subsection{Paste preparation}

The primary paste formulation consisted of microcrystalline cellulose powder (Avicel PH101, FMC Corporation, Ireland: solid density $1540 \mathrm{~kg} \mathrm{~m}^{-3}$; moisture content $\sim 3 \mathrm{wt} \%$; particle size measured by Coulter LS230 laser diffraction, ranging from 2 to $260 \mu \mathrm{m}$ and Sauter mean diameter of $49.1 \mu \mathrm{m}$ [21]) combined with reverse osmosis water. A second formulation featured a mixture of MCC/lactose in water. $\alpha$-lactose monohydrate (Sigma-L3625, Sigma Aldrich, UK: solid density $1540 \mathrm{~kg} \mathrm{~m}^{-3}$; particle sizes measured by laser diffraction 0.5 to $215 \mu \mathrm{m}$ and mean circle-equivalent diameter of $44.1 \mu \mathrm{m}$ ) was mixed with MCC and the water content optimised by trial and error at $50 \mathrm{wt} \%$.

Calcium carbonate (Pumex UK Ltd, Newcastle-under-Lyme, Staffordshire: calcite; solid density $2710 \mathrm{~kg} \mathrm{~m}^{-3}$ ) was used as a model 'hard', insoluble API within the plastic MCC-water matrix. Laser diffraction indicated a biomodal particle size distribution ranging from 0.2 to $40 \mu \mathrm{m}$, with modes at $0.9 \mu \mathrm{m}$ and $11 \mu \mathrm{m}$ and a Sauter mean diameter $4.2 \mu \mathrm{m}$. Calcium carbonate was added to give model API contents of 5, 10 and $15 \mathrm{wt} \%$ to the primary formulation. The MCC/water ratio was kept constant so that the rheology of the wet mass was determined principally by the presence of the calcite particulates. 
Table 2 summarises the paste formulations and the labelling used.

\subsection{Extrusion}

Paste extrusion was performed using a Zwick/Roell Z50 strain frame configured to operate as a ram extruder as described by Zhang et al. [21]. The same configuration was used for paste characterisation and extrudate generation. A stainless steel ram fitted with a polyether ether ketone (PEEK) tip forced paste from a cylindrical barrel (internal diameter $D_{\mathrm{b}}$ ) through a concentric, square entry die. The barrel and dies were all constructed from 316 stainless steel. The crosshead position and force on the ram were monitored continuously with resolution $\pm 1 \mu \mathrm{m}$ and $\pm 0.1 \mathrm{~N}$, respectively. The ram force was expressed as the mean extrusion pressure, $P_{\mathrm{ex}}$, from $P_{\mathrm{ex}}=4 \times$ force $/ \pi D_{\mathrm{b}}^{2}$.

A paste charge (approximately $90 \mathrm{~g}$ for E-S testing) was loaded into the barrel, pre-compacted to $0.9 \mathrm{MPa}$ to consolidate the material, then extruded at a steady ram velocity. The extrudate velocity, $V$, along the die land (internal diameter $D$ ) was calculated by assuming conservation of volume, so that $V=$ ram velocity $\times D_{\mathrm{b}}{ }^{2} / D^{2}$. The extrusion pressure increased at the start of a run as the die land filled and static zones formed within the barrel, and remained steady until the end of the run (ram travel: $130 \mathrm{~mm}$ for extrudate generation runs; $75 \mathrm{~mm}$ for characterisation tests). $P_{\mathrm{ex}}$ was taken as the average value between ram displacements of $D_{\mathrm{b}}$ and $2 D_{\mathrm{b}}$. Data and extrudates generated from runs which did not give steady profiles were discarded.

Extrudate generation runs employed one die land $(D=3.5 \mathrm{~mm}$, length $L=16 \mathrm{~mm})$ and a ram velocity of $1 \mathrm{~mm} \mathrm{~s}^{-1}$. The same barrel $\left(D_{\mathrm{b}}=25 \mathrm{~mm}\right)$ was used for paste characterisation, using a series of $3.5 \mathrm{~mm}$ diameter die lands of lengths $4,16,32$ and $42 \mathrm{~mm}$, and ram velocities $(0.5$, $1.0,3.0,5.0 \mathrm{~mm} \mathrm{~s}^{-1}$, corresponding to $V=26,51,153$ and $255 \mathrm{~mm} \mathrm{~s}^{-1}$ ). The $P_{\text {ex }}$ data set was then fitted to the Benbow-Bridgwater equation [17]:

$$
P_{\mathrm{ex}}=2 \underbrace{\left(\sigma_{\mathrm{Y} 0}+\alpha V^{m}\right)}_{\sigma_{\mathrm{Y}}} \ln \frac{D_{\mathrm{b}}}{D}+4 \underbrace{\left(\tau_{\mathrm{W} 0}+\beta V^{n}\right)}_{\tau_{\mathrm{W}}} \frac{L}{D}
$$

where the bulk yield strength, $\sigma_{\mathrm{Y}}$, and the wall shear stress, $\tau_{\mathrm{w}}$, are treated as a combination of two contributions: $\sigma_{\mathrm{Y} 0}$ and $\tau_{\mathrm{w} 0}$ are the bulk yield strength and wall shear stress both at zero extrudate velocity, respectively. $\alpha$ and $\beta$ are velocity factors with power law indices $m$ and $n$, 
respectively. Further discussion of the approach is given in [22]. High speed camera testing was also performed with $3.5 \mathrm{~mm}$ diameter extrudates. All tests were performed at room temperature $\left(20-25^{\circ} \mathrm{C}\right)$ and relative humidity normally between $32-58 \%$.

The density of the paste was calculated at the end of the precompaction stage. Precompaction was performed with a blank die (i.e. a flat platen) in place. The mass and height of the compacted paste billet were measured. The procedure was repeated over 15 times for each paste and densities are reported with a standard deviation of $10 \mathrm{~kg} \mathrm{~m}^{-3}$ (see Table 2).

Paste and extrudate water contents were determined by measuring the mass of a sample before and after drying in a vacuum oven at $40^{\circ} \mathrm{C}$ and $100 \mathrm{mbar}$ for $24 \mathrm{~h}$. Separate tests indicated that this was sufficient time for the dry mass to reach a steady value. The results confirmed that loss of water from the paste due to evaporation was minimal.

\subsection{Spheronisation}

Spheronisation was performed using the same Caleva Spheroniser 120 (Caleva Process Solutions Ltd, UK) employed by Bryan et al. [1] and Lau et al. [15]. This featured a $120 \mathrm{~mm}$ diameter 316 stainless steel cross-hatched friction plate with square-based truncated pyramidal elements on a square pattern (pitch $1.40 \mathrm{~mm}$, height $0.86 \mathrm{~mm}$ and width at top $0.50 \mathrm{~mm}$ ). Detailed spheronisation studies were performed at a rotational speed, $\omega$, of $1200 \mathrm{rpm}$ (confirmed by tachometry), corresponding to a plate rim velocity, $\omega R$, of $7.5 \mathrm{~m} \mathrm{~s}^{-1}$. Bryan et al. [1] used the same speed in their study.

Extrudates were prepared for spheronisation following the procedure reported by Bryan et al. [1]. $N_{0}=20$ identical cylindrical extrudates $\left(b=D=3.5 \mathrm{~mm}, l_{0}=21.5 \mathrm{~mm}\right)$ were cut from freshly extruded material, weighed and placed in the spheroniser. The paste charge was subjected to rotation for a set time, then removed, passed over a $2.36 \mathrm{~mm}$ sieve to remove any fines or fragments and re-weighed to determine the mass lost due to the generation of fines and evaporation of water. The pellets were also weighed after size and shape analysis to check that the amount of water lost via evaporation was minimal.

\subsection{Pellet size and shape analysis}

Following sieving, the pellet sample was spread out on black photographic paper and each 
pellet was photographed individually with a calibrated digital microscope (USB Digital Microscope $400 \times$, Maplin, UK). The images were analysed using the MATLAB ${ }^{\circledR}$ code developed by Bryan et al. [1], which calculated a range of shape parameters. Only one parameter was considered here, namely the aspect ratio (see Section 2.1 above), as this was sufficient to track the approach to the spheronisation end time. Measures such as the 'dumbbellity' parameter developed by Bryan et al. [1] are useful for monitoring pellet shape evolution in the early stages of rounding but are relatively insensitive as $t \rightarrow t_{\text {end }}$.

\subsection{High speed camera analysis}

Videos of the spheronisation of paste extrudates were taken with a Photron Fastcam SA 3 camera (Photron Europe, West Wycombe, UK) fitted with a Sigma 24-70 mm F2.8 lens (Sigma Corp, New York, USA) mounted vertically above the friction plate. The bed was illuminated using a fibre-optic halogen lamp (OSL2, Thorlabs, New Jersey, USA). Images of $512 \times 512$ pixels were collected at $1000 \mathrm{fps}$, acquiring $5 \mathrm{~s}$ of real time data for each episode.

Analysis of the high speed camera recordings was performed for a selection of pellets by manually tracking their motion during the initial stages of spheronisation using ImageJ image analysis software (NIH, Maryland, USA). The experiments incorporated 20 rods of freshly cut extrudate, each $3.5 \mathrm{~mm}$ in diameter and $21.5 \mathrm{~mm}$ long, produced from the extrusion of paste $M$. The results are presented in terms of two stages, viz. an initial extrudate rod breakage stage, followed by a rod fragment rounding stage.

\section{Estimation of pellet velocity}

The software was used to determine the location of a given point on each pellet (with coordinate origin located at the centre of the friction plate) in sequential video frames separated by a time step $\Delta t$. A scaling factor of $0.28 \mathrm{~mm} /$ pixel was used (found by taking several measurements of the $120 \mathrm{~mm}$ friction plate diameter), and the Cartesian locations were converted into polar coordinates. This allowed the instantaneous particle velocity, decomposed into radial and azimuthal components, to be estimated. The image analysis also indicated that the friction plate took approximately $90 \mathrm{~ms}$ to accelerate to the pre-set rotational speed of $1200 \mathrm{rpm}$.

High speed camera analysis would ideally provide an estimate of the frequency of collisions 
and the impact velocity distribution of a typical pellet during the breakage and rounding stages. However, there was insufficient time to develop the sophisticated pellet tracking code necessary to achieve this. One issue that must be overcome is the use of time-discrete position data to compute pellet velocities, which generates errors that are highly sensitive to the time step $\Delta t$ employed (which was limited by the video capture frame rate). The high velocities in spheronisation can also lead to uncertainty in tracking any given pellet throughout the course of the analysis, which any tracking code would have to take into account. The manual tracking results presented here serve to illustrate the general trends in velocity observed.

\section{Results and Discussion}

\subsection{Paste density and yield strength}

Calcium carbonate is more dense than the MCC-water paste matrix (M). The data in Table 2 suggest a linear increase in density with mass fraction of $\mathrm{CaCO}_{3}$. The values are close to those estimated assuming the carbonate to be insoluble. The MCC-lactose paste (ML) is also more dense than $\mathrm{M}$, which is attributed to the dissolution of some lactose into the liquid phase (the solid densities of MCC and lactose are similar).

Paste characterisation tests gave linear pseudo-Bagley plots, where $P_{\text {ex }}$ is plotted against $L / D$, an example of which is shown in Figure 1. This indicates that the wall shear stress in the die land is not sensitive to extrusion pressure, which is one of the assumptions in the BenbowBridgwater approach (Equation (16)). The intercept on the ordinate gives an estimate of $\sigma_{\mathrm{Y}}$ at test velocity $V$, while the gradient gives $\tau_{\mathrm{w}}$. Figure 2(a) shows that $\sigma_{\mathrm{Y}}$ for any given paste was insensitive to $V$, indicating that these pastes can be treated as pseudo-plastic materials. Similar findings were obtained for related MCC/water pastes by Zhang et al. [21]. There is thus little effect of deformation rate on the plastic yield strength, and $\sigma_{\mathrm{Y}}$ in the dimensional analysis is set equal to $\sigma_{\mathrm{Y} 0}$. The paste containing lactose (ML) was also pseudo-plastic, with a yield strength about twice that of the MCC-water paste.

The wall shear stress data in Figure 2(b) show a noticeable effect of $V$. Fitting a power law model to the data (see Equation (16)) gave $n$ values ranging from 0.34 to 0.55 . The $\tau_{\mathrm{w}}$ values at a given value of $V$ generally increase with $\mathrm{CaCO}_{3}$ content, but the increase is not as large as those observed for $\sigma_{\mathrm{Y}}$ (Figure 2(a)). Furthermore, for a given paste formulation, the values of wall shear stress are consistently smaller than the bulk yield stress, which supports the 
assumption of wall slip (deformation primarily occurring at the wall) in Equation (16). The lactose-containing paste (ML) exhibits similar behaviour to paste $\mathrm{M}$, albeit with a larger sensitivity to $V(n=0.55 c f .0 .34$ for paste $\mathrm{M})$.

The effect of $\mathrm{CaCO}_{3}$ content on $\sigma_{\mathrm{Y}}$ is presented in Figure 3. Whereas the density increases linearly with increasing mass fraction of carbonate, the yield strength increases exponentially. The linear relationship between $\log _{10} \sigma_{\mathrm{Y}}$ and carbonate content is similar to that reported for clay-water-gravel mixtures by Kumar and Muir Wood [23]: in this context, the $\mathrm{CaCO}_{3}$ particles are analogous to gravel particles, acting as hard inclusions within a plastic matrix.

Increasing the carbonate content of the paste resulted in the generation of fragments during spheronisation, which subsequently rounded to give a bimodal pellet size distribution. These pellets passed through the $2.36 \mathrm{~mm}$ mesh and are not considered in the following discussion of pellet shape. Bryan et al. [9] also reported a pattern for stiffer pastes (in their case, resulting from high shear mixing) to give bimodal pellet size distributions.

\subsection{Evolution of pellet shape}

The aspect ratio was used to gauge the spheronisation end time, $t_{\text {end }}$, and a systematic procedure was required to identify this quantity. Figure $4(a)$ shows a data set obtained for spheronisation of paste $\mathrm{M}$ reported by Bryan et al. [1], with $A R$ plotted against $t$ on a $\log _{10}$ scale. Note that there is some uncertainty in the $t_{\text {end }}$ value reported, since they judged the end time by eye, and did not use the systematic approach reported here. Fewer interrupted tests were conducted in the current study as the objective was primarily to identify $t_{\text {end }}$. $A R$ reaches an average value of 0.95 , which lies comfortably above the threshold of 0.8 for pharmaceutical applications reported by Chopra et al. [24].

Two regions are evident. In the first region, $A R$ approaches a final value, labelled $A R_{\text {end, }}$ almost asymptotically, and in the approach to $A R_{\text {end }}$ follows a linear dependency on $\log _{10} t$. One could equally well plot $A R$ against $\ln t$; a $\log _{10}$ scale is used here as it conveys the timescale more conveniently. In the second region, the average value of $A R$ is constant and there is some reduction in the width of the distribution; this region was used to identify $A R_{\text {end }}$, the value of which could vary with paste formulation. This pattern, which was also reported by Lau et al. [15] and is evident in data reported by other workers (see Supplementary 
Figures S1, S2 and S3), was observed with all five pastes tested in this study. The data obtained for the MCC-lactose water paste are presented in Figure 4(b) with construction lines showing $A R_{\text {end }}$ and $t_{\text {end }}$.

Loci showing the trends predicted by the simple models (Equations (14) and (15)) are plotted alongside the experimental data in Figure 4(a). Each model has two adjustable parameters, an initial value $\left(A R_{\text {end }}-A R\right)_{0}$, which has been set to $(1.0-0.65)=0.35$, and a rate constant $k$. (Note that if an extrudate broke into three cylinders of equal length, then the initial $A R$ values of the fragments would be approximately 0.5.) Both models follow the general trend in the data, but the variation in the $A R$ values prevents any differentiation between the two models being made. Moreover, there is no evidence to suggest that the mechanism remains first or second order throughout the rounding process.

The following systematic procedure was developed to quantify $t_{\text {end }}$. An initial estimate was obtained by monitoring the progress of spheronisation by eye. A series of spheronisation tests were then conducted, bracketing this initial estimate. The $A R$ distributions were calculated and plotted against $t$ on a $\log _{10}$ scale (see Figure 5). $A R_{\text {end }}$ was identified as described above. $t_{\text {end }}$ was taken as the midpoint between the last datum where $A R<A R_{\text {end }}$ and the first where $A R=A R_{\text {end }}$. An alternative method, of fitting a line of the form $A R=\log _{10} t+c$ through the data in the first region and projecting it to meet $A R_{\text {end }}$, gave estimates of $t_{\text {end }}$ which agreed with those obtained using the above method to within the uncertainty of the experimental data. The values of $A R_{\text {end }}$ and $t_{\text {end }}$ are reported in Table 2.

\subsection{Dimensional analysis}

Equations (6) and (7) indicate that both $A R_{\text {end }}$ and $\omega t_{\text {end }}$ will depend on the ratio of the bulk yield strength to the kinetic energy of the pellet per unit volume, represented by the dimensionless group $\sigma_{\mathrm{Y}} / \rho(\omega R)^{2}$. Figure 6 shows linear relationships in both cases for the MCC-based series of pastes with added carbonate. $A R_{\text {end }}$ decreases with increasing $\sigma_{\mathrm{Y}} / \rho(\omega R)^{2}$, which can be interpreted as the kinetic energy dissipated in successful collisions being unable to cause significant change in the sphericity of the pellet. The change in $A R_{\text {end }}$, of 0.05 , for a three-fold increase in $\sigma_{\mathrm{Y}} / \rho(\omega R)^{2}$ is appreciable, given that $A R_{\text {end }}$ should ideally lie between 0.8 and 1.0. The fitted linear trend line is 


$$
A R_{\mathrm{end}}=0.988-0.0089 \frac{\sigma_{\mathrm{Y}}}{\rho(\omega R)^{2}}
$$

Referring to Figure $6(b), \omega t_{\text {end }}$ displays a six-fold increase for a three-fold increase in $\sigma_{\mathrm{Y}} / \rho(\omega R)^{2}$ :

$$
\omega t_{\mathrm{end}}=4100 \frac{\sigma_{\mathrm{Y}}}{\rho(\omega R)^{2}}-8000
$$

The results for the MCC-lactose (ML) paste and the MCC-only (M) paste reported by Bryan et al. [1] lie in similar regions to the above series. The ML paste is expected to feature different friction coefficients, so close agreement is not expected. Bryan et al. [1] employed a similar system (device, geometry, velocity) to generate their extrudates but did not characterise their MCC-water paste. They did, however, report a mean extrusion pressure of $4 \mathrm{MPa}$ for their material, which is higher than that observed in this work, of approximately 2.5 MPa. This is expected to increase the yield strength of the paste, which would shift the data points from their plotted positions (which were calculated using the same $\sigma_{\mathrm{Y}}$ value as that for paste $\mathrm{M}$ ) to the right on Figure 6, giving better agreement with the present work. There is also some uncertainty to the $t_{\text {end }}$ value reported by Bryan et al. [1] in Figure $6(b)$, since they judged this value by eye.

Since the linear relationships given by Equations (17) and (18) have now been established, it follows that the data can be compared using the form suggested by Equation (8). The individual evolution profiles (plots of $A R$ against $t$ ) are displayed alongside each other in Figure 7 using $A R / A R_{\text {end }}$ and $t / t_{\text {end }}$ axes. The scaling reduces the data sets to a common form, including that collected with the MCC-lactose (ML) paste. The good agreement of the experimental data indicates that the estimate of paste yield strength, derived from ram extrusion experiments, is a reliable indicator of this property. These results (and those in the Supplementary Figures S1, S2 and S3) indicate that rounding of pellets during spheronisation follows a rate law of the form where the approach to sphericity is proportional to $\log _{10} t$. This finding has potential application in formulation development as it could be used to reduce the number of tests required to determine $t_{\text {end }}$. The reason for the form of the rate law remains unresolved. The simple models presented here (Equations (14) and (15)) are not able to describe the process over the complete timescale, which is an indicator that there are a number of mechanisms active. Elucidating the contribution of each can be achieved by 
techniques such as DEM. Elucidating the contribution of each can be achieved by techniques such as population balance modelling, with phenomenological insight provided by DEM.

\subsection{High speed camera results}

\subsubsection{Extrudate rod breakage stage}

During start-up of the spheronisation test $(0<t<0.1 \mathrm{~s})$, visual inspection of the high speed camera footage indicated that the acceleration of the friction plate to the tip speed of $7.5 \mathrm{~m} \mathrm{~s}^{-1}$ caused the extrudate rods to move radially towards the wall, initially via a rolling mechanism, followed by sliding along the friction plate. Initial rod-wall collisions rarely resulted in rod breakage. Upon reaching the wall, the majority of the rods remained prone on the friction plate and travelled along the wall periphery; typical azimuthal velocities of these rods were estimated to be approximately 15-fold lower than the tip speed of the friction plate.

Changes in rod orientation to an upright position were induced mainly by rod-rod collisions, which were seen to give rise to rod breakage either directly on impact (rarely observed) or indirectly via the acceleration of rod ends caused by a near perpendicular collision with the moving friction plate or the static spheroniser wall. The rods generally broke into three fragments (usually of unequal lengths) following two collisions; this observation agrees with the results of Bryan et al. [1] and Lau et al. [15] for spheronisation tests with a charge of 20 rods, which produced approximately 60 rounded pellets. The generation of these shorter rods was then seen to initiate a chain of breakage events, leading to the breakage of all the initial rods in under $3 \mathrm{~s}$. This corresponds to the breakage stage occupying the first $5 \%$ of the spheronisation time and is consistent with the findings of Bryan et al. [1], who showed that the total number of pellets and their characteristic size remained constant after $t / t_{\text {end }} \sim 0.05$.

An example of video footage is provided as Supplementary Video 1. The clip is in slow motion, covering $1.5 \mathrm{~s}$ of real time, and shows periods of extrudate rod breakage (taken at $t \sim 0.6 \mathrm{~s}$ ) and pellet rounding (taken at $t \sim 20 \mathrm{~s}$ ). By the rounding stage, the initial batch of 20 extrudate rods had broken to give approximately 60 pellets and several dumb-bell/ellipse shaped bodies are evident. 
Figure 8 shows an example of the radial and azimuthal velocities of an extrudate rod tracked during the breakage stage $(t<0.6 \mathrm{~s}$ in this case). The average azimuthal velocity is approximately $1.0 \mathrm{~m} \mathrm{~s}^{-1}$, which at any given time is smaller than the azimuthal velocity of the friction plate calculated at the same radial position as the rod (between 5.5 and $7.5 \mathrm{~m} \mathrm{~s}^{-1}$ ). This difference in velocity is due to the rolling/sliding of the rod on the friction plate, or when the rod loses contact with the plate. The radial velocity of the rod varies from zero (i.e. static) with excursions up to $\pm 0.3 \mathrm{~m} \mathrm{~s}^{-1}$ throughout the period. The rod is seen to accelerate and decelerate at various times, which is due to its interaction with the friction plate, other rods/fragments, and/or the wall.

\subsubsection{Rod fragment rounding stage}

After the initial rod breakage, the rounding mechanism was found to depend on the rod fragment length. Shorter fragments were mainly seen to move along the friction plate periphery at azimuthal velocities of up to $5 \mathrm{~m} \mathrm{~s}^{-1}$. Sometimes they were seen to round directly into spheres without passing through the dumb-bell stage. By contrast, longer fragments were often observed to travel in a slower moving plane in the region above the friction plate (with azimuthal velocities of the fragments estimated as $\sim 1 \mathrm{~m} \mathrm{~s}^{-1}$ ). When any fragments made contact with the plate, they interacted with the surface in a somersault-like motion (from end to end), causing them to round off into dumb-bells and produce fines via particle attrition. Collisions with other fragments and the spheroniser wall, resulting in sudden acceleration and deceleration (i.e. momentum transfer events), were also seen to play an important role in rounding.

Figure 9 presents an example of the radial and azimuthal velocities of a rod fragment during the rounding stage $\left(20.0<t<20.2 \mathrm{~s}\right.$ in this case, corresponding to $\left.0.44<t / t_{\text {end }}<0.45\right)$. Comparing this velocity history with that during the breakage stage (Figure 8), the average azimuthal velocity of the fragment during the rounding stage is higher (approximately 2.5$3.0 \mathrm{~m} \mathrm{~s}^{-1}$ ). This may be due to a stronger interaction between the fragment and the friction plate for these smaller pellets, as described above. The radial velocity fluctuates around zero. The fragment is once again seen to accelerate and decelerate at various times as a result of interactions with the friction plate, other fragments and/or the wall. There is a period where the fragment experiences fewer velocity fluctuations (regime (2)), which corresponds to it moving with a cluster of other fragments in a region above the friction plate. Collisions during 
this period are unlikely to result in significant change in shape.

Comparing Figures 8 and 9 also emphasises the importance of the time step chosen for data analysis. The smaller $\Delta t$ value in Figure 9, of $2 \mathrm{~ms}$, allows higher resolution of any velocity fluctuations.

These results are consistent with the velocity distributions in beds of pellets measured using particle image velocimetry (PIV) by Koester and Thommes [14]. They studied the spheronisation of $1 \mathrm{~mm}$ diameter MCC-lactose extrudates generated by a twin-screw extruder fitted with a multi-holed die plate (length to diameter ratio of 2.5) and subsequently spheronised on a $300 \mathrm{~mm}$ diameter friction plate operating at rotational speeds of 500 to $1000 \mathrm{rpm}$. Their batches were considerably larger than those employed in this work and the pellets formed a toroidal bed several $\mathrm{cm}$ wide adjacent to the rim of the friction plate. They reported surface pellet velocities ranging from approximately $1 \mathrm{~m} \mathrm{~s}^{-1}$ on the top of the toroidal bed to $2 \mathrm{~m} \mathrm{~s}^{-1}$ at its inner edge, in contact with the friction plate, for a plate tip speed $(R \omega)$ of $11.8 \mathrm{~m} \mathrm{~s}^{-1}$. The pellets at the inner edge were free to move in all directions, including away from the friction plate. This freedom of motion was marked by the granular temperature being highest in this region (the granular temperature is a measure of the fluctuation in velocity). The velocities on the top of the bed, beyond the inner edge, were lower owing to multiple collisions with other pellets, and were accompanied by a low granular temperature (approaching zero). The characteristic pellet velocities were thus substantially lower than the tip speed (as in Figure 8), and the data in Figures 8 and 9 can be related to the inner edge of Koester and Thommes' toroidal bed.

Data from the high-speed camera analysis suggest that the less chaotic motion of pellets during rounding is the reason why this stage is the rate-determining step in the spheronisation process. The velocity of the pellets does not scale with the rim speed in a simple manner. Moreover, the kinetic energy available for transfer during pellet collision events during the rounding stage is smaller as the pellet velocities are noticeably lower than the spheroniser tip speed. This was observed as the pellet motion changed from frequent contact with the spheroniser friction plate (pellet azimuthal velocity of approximately $4 \mathrm{~m} \mathrm{~s}^{-1}$ ) to often travelling in a region above the plate (either individually or as a pellet cluster) at lower azimuthal velocities of $\sim 1 \mathrm{~m} \mathrm{~s}^{-1}$. Pellet-plate collisions were comparatively brief, and served 
to project pellets back into the air. The dimensionless group $\sigma_{\mathrm{Y}} / \rho(\omega R)^{2}$ will overestimate the efficiency of collisions as the kinetic energy per unit volume of an individual pellet will be a fraction of $1 / 2 \rho(\omega R)^{2}$.

Similar behaviour was observed in tests performed at $1800 \mathrm{rpm}$ (corresponding to a tip speed of $11.3 \mathrm{~m} \mathrm{~s}^{-1}$ ) using the same paste and initial load of extrudate. The magnitude of velocity of the rod was expected to increase with the spheronisation speed; however, over the short preliminary tracking of $0.2 \mathrm{~s}$ duration, no notable changes were recorded.

These findings suggest that future efforts in this area should focus on whether detailed modelling of pellet motion, such as the DEM studies by Al-Harbi et al. [12], can replicate the patterns and magnitudes evident in these velocity data.

\subsubsection{Influence of paste formulation}

High-speed camera recordings of paste ML and MA15 indicated similar spheronisation mechanisms to those reported above. However, with MA15, the extrudate rods were initially subject to noticeable abrasion by contact with the friction plate, thus producing a higher proportion of fines and fragments which rounded into smaller, spherical pellets. The latter passed through the mesh used to remove fines and would otherwise give a bimodal pellet size distribution similar to that reported by Bryan et al. [9]. Both of these observations can be attributed to the drier, less cohesive nature of the paste with relatively high carbonate content.

\subsection{Topics for further work}

A dimensional analysis of E-S has been developed and validated for two somewhat similar paste materials. Further work is recommended to demonstrate the applicability of the dimensionless correlation to other widely used pharmaceutical paste formulations, as well as investigating the effect of process variables such as amount of extrudate, spheroniser friction plate radius and rotational velocity. It would also be of interest to monitor the evolution of pellet porosity, since this is indicative of the forces of compaction experienced by the pellets and so could improve the mechanistic understanding of spheronisation and scale-up to different tip speeds. The final porosity of the pellets also dictates their mechanical strength, and so is likely to be a useful measure of final product quality. 
More reliable methods of extracting pellet velocity data from high-speed camera imaging should also be developed with the aim to: $(i)$ obtain larger datasets of pellet velocities for the purpose of statistical analysis; and (ii) investigate the types of collisions occurring at the different stages during spheronisation, as well as their frequencies and magnitudes of energy transfer. These would require the ability to track pellets automatically and to output the corresponding velocity vector field and energy map for further analysis.

\section{Conclusions}

The mechanisms of extrusion-spheronisation were investigated for MCC/water-based pastes using the techniques of ram extrusion and pellet shape evolution as reported by Bryan et al. [1]. Spheronisation tests using calcium carbonate to mimic the addition of a hard, active pharmaceutical ingredient to the paste matrix demonstrated the same mechanistic steps for the process, where the initial break-up of extrudates occurred rapidly with most of the spheronisation time being used to round the pellets. Two simple rate models that considered an 'approach to sphericity' were proposed to describe the rounding behaviour of pellets, but verification was hindered due to uncertainty in experimental data.

The bulk yield strength of the pastes was quantified using the Benbow and Bridgwater paste characterisation method [17]. Addition of calcium carbonate was shown to increase the yield strength of the paste, which resulted in longer spheronisation times and less spherical pellet shape (here quantified by the aspect ratio, $A R$ ). The effect of carbonate addition on the end properties of the system ( $A R_{\text {end }}$ and $\left.t_{\text {end }}\right)$ was found to be correlated with the dimensionless ratio of paste bulk yield strength to a collision energy per unit volume. Non-dimensionalising the experimental shape evolution data unified all the datasets, and the resulting $A R / A R_{\text {end }}$ against $t / t_{\text {end }}$ profile was found to follow a linear-logarithmic trend towards an asymptote. An investigation with a different paste composition, namely MCC/lactose/water, confirmed the validity of the dimensionless correlation in predicting the evolution of pellet shape.

Quantitative information extracted from high-speed camera imaging of spheronisation of MCC-water pastes indicated that the pellet velocity was often much lower than the spheroniser plate tip speed, viz. $\sim 1 \mathrm{~m} \mathrm{~s}^{-1}$ compared to $7.5 \mathrm{~m} \mathrm{~s}^{-1}$, respectively. This appeared to have limited effect on the timescale for the initial rapid breakage of extrudates. In contrast, the 
relatively low velocity of pellets and the reduced efficiency of collisions during the rounding stage provided evidence for this being the rate-determining step. Additional evidence was found in terms of qualitative observations of the less chaotic motion exhibited by pellets in the rounding stage, attributed to their corresponding increase in sphericity. Tests with carbonate-containing pastes showed that the same mechanisms were controlling spheronisation, while the use of drier and hence less cohesive pastes was seen to inflate the production of fines.

\section{Acknowledgements}

Microcrystalline cellulose for this final year student research project was kindly provided by MSD Devlab, Hoddesdon, UK. Support for M.P. Bryan from CERATIZIT GmbH and Sandvik Hyperion is gratefully acknowledged. 


\section{Nomenclature}

\section{Roman}

\begin{tabular}{|c|c|c|}
\hline$A R, A R_{\text {end }}$ & Aspect ratio, final value & - \\
\hline$b$ & Minor axis length (breadth) of pellet & $\mathrm{m}$ \\
\hline$c$ & A constant & - \\
\hline$D$ & Extrusion die diameter, extrudate diameter & $\mathrm{m}$ \\
\hline$D_{\mathrm{b}}$ & Extrusion barrel diameter & $\mathrm{m}$ \\
\hline$g$ & Gravitational acceleration & $\mathrm{m} \mathrm{s}^{-2}$ \\
\hline$H$ & Characteristic size of spheroniser friction plate elements & $\mathrm{m}$ \\
\hline$k$ & A constant & - \\
\hline$k_{1}$ & Rate constant, Equation (11) & $\mathrm{s}^{-1}$ \\
\hline$k_{2}$ & Rate constant, Equation (13) & $\mathrm{s}^{-1}$ \\
\hline$l$ & Major axis length of pellet & $\mathrm{m}$ \\
\hline$l_{0}$ & Initial rod length & $\mathrm{m}$ \\
\hline$L$ & Extrusion die land length & $\mathrm{m}$ \\
\hline$m$ & Power law index, Equation (16) & - \\
\hline$n$ & Number of collisions; Power law index, Equation (16) & - \\
\hline$N, N_{0}$ & Number of pellets, initial value & - \\
\hline$P_{\mathrm{ex}}$ & Mean extrusion pressure & $\mathrm{Pa}$ \\
\hline$R$ & Radius of spheroniser friction plate & $\mathrm{m}$ \\
\hline$t$ & Time & s \\
\hline$t_{\text {end }}$ & Time to complete spheronisation & $\mathrm{s}$ \\
\hline$u$ & Pellet velocity & $\mathrm{m} \mathrm{s}^{-1}$ \\
\hline$u_{\mathrm{r}}$ & Pellet velocity, radial direction & $\mathrm{m} \mathrm{s}^{-1}$ \\
\hline$u_{\theta}$ & Pellet velocity, azimuthal direction & $\mathrm{m} \mathrm{s}^{-1}$ \\
\hline$V$ & Extrudate velocity in die land & $\mathrm{m} \mathrm{s}^{-1}$ \\
\hline$y$ & A measure of pellet sphericity, Equation (9) & - \\
\hline
\end{tabular}




\section{Greek}

$\alpha$

$\beta$

$\mu_{\mathrm{p}}$

$\mu_{\mathrm{s}}$

$\rho$

$\sigma_{\mathrm{Y} 0}$

$\sigma_{\mathrm{Y}}$

$\tau_{\mathrm{w}}$

$\tau_{0}$

$\omega$

Acronymns

API

E-S

MCC

PEEK

PIV
Bulk yield strength velocity factor, Equation (16)

Wall shear stress velocity factor, Equation (16)

Friction coefficient, pellet-pellet

Friction coefficient, pellet-spheroniser surface

Density

Bulk yield strength at zero extrudate velocity, Equation (16)

Bulk yield strength

Wall shear stress

Wall shear stress at zero extrudate velocity, Equation (16)

Spheroniser rotational speed
$\mathrm{Pa}\left(\mathrm{s} \mathrm{m}^{-1}\right)^{m}$

$\mathrm{Pa}\left(\mathrm{s} \mathrm{m}^{-1}\right)^{n}$

$\mathrm{kg} \mathrm{m}^{-3}$

$\mathrm{Pa}$

$\mathrm{Pa}$

$\mathrm{Pa}$

$\mathrm{Pa}$

$\mathrm{s}^{-1}$

Active pharmaceutical ingredient

Extrusion-spheronisation

Microcrystalline cellulose

Polyether ether ketone

Particle image velocimetry 


\section{References}

[1] Bryan, M.P., Atherton, L.N., Duffield, S., Rough, S.L. and Wilson, D.I. (2015a) Stages in spheronisation: evolution of pellet size and shape during spheronisation of microcrystalline cellulose-based paste extrudates, Powder Tech., 270, 163-175.

[2] Haring, A., Vetchy, D., Janovska, L., Krejcova, K. and Rabiskova, M. (2008) Differences in characteristics of pellets prepared by different pelletization methods, Drug Dev. Ind. Pharm., 34(3), 289-296.

[3] Vervaet, C., Baert, L. and Remon, J.P. (1995) Extrusion-spheronisation: A literature review, Int. J. Pharm., 116(2), pp. 131-146.

[4] Wilson, D.I. and Rough, S.L. (2007) Extrusion-Spheronisation, in Granulation, eds Salman, A.D. and Hounslow, M.J., Elsevier, Amsterdam, 189-210.

[5] Rowe, R.C. (1985) Spheronization: a novel pill-making process?, Pharm. Int., 6, 119123.

[6] Baert, L., Vermeersch, H., Remon, J.P., Smeyers-Verbeke, J. and Massart, D.L. (1993) Study of parameters important in the spheronisation process. Int. J. Pharm. 96, 225229.

[7] Liew, C.V., Chua, S.M. and Heng, P.W.S. (2007) Elucidation of spheroid formation with and without the extrusion step, AAPS PharmSciTech, 8, 1-10.

[8] Koester, M., Willemsen, E., Krueger, C. and Thommes, M. (2012) Systematic evaluations regarding interparticular mass transfer in spheronisation, Int. J. Pharm., 431(1-2), 84-89.

[9] Bryan, M.P., Kent, M.D., Rickenbach, J., Rimmer, G., Wilson, D.I. and Rough, S.L. (2015b) The effect of mixing on the extrusion-spheronisation of a micro-crystalline cellulose paste, Int. J. Pharm., 479(1), 1-10.

[10] Sinka, C.I. (2011) A first order numerical study of the spheronisation process, Powder Tech., 206, 195-200.

[11] Sinka, C.I. (2015) A model for the deformation of an ellipsoid subject to a large number of successive impacts with special reference to spheronisation, Powder Tech., 270, 592-598.

[12] Al-Harbi, A., Elmsahli, M. and Sinka, C.I. (2015) Discrete element modelling of particle collisions in granular flows with special reference to spheronisation, Proc. Granulation Conference, Sheffield, paper 9.

[13] Bouffard, J., Cabana, A., Chaouki, J. and Bertrand, F. (2012) Experimental investigation of the effect of particle cohesion on the flow dynamics in a spheronizer, AIChEJ, 59(5), 1491-1501.

[14] Koester, M. and Thommes, M. (2013) Analysis of particle kinematics in spheronisation via particle image velocimetry, Eur. J. Pharm. Biopharm., 83, 307-314.

[15] Lau, C.L.S., Yu, Q., Lister, V.Y., Rough, S.L., Wilson, D.I. and Zhang, M. (2014) The evolution of pellet size and shape during spheronisation of an extruded microcrystalline cellulose paste, Chem. Eng. Res. Des, 92, 2413-2424.

[16] Adams, M.J., Lawrence, C.J., Urso, M.E.D. and Rance, J. (2004) Modelling collisions of soft agglomerates at the continuum length scale, Powder Tech., 140, 268-279. 
[17] Benbow, J.J. and Bridgwater, J. (1993) Paste Flow and Extrusion, Clarendon Press, Oxford.

[18] Fielden, K.E., Newton, J.M. and Rowe, R.C. (1992) The influence of lactose particle size on spheronisation of extrudate processed by a ram extruder, Int. J. Pharm., 81, 205-224.

[19] Heng, P.W.S. and Koo, O.M.Y. (2001) A study of the effects of the physical characteristics of microcrystalline cellulose on the its performance in extrusionspheronisation, Pharm. Res., 18, 480-487.

[20] Macritchie, K.A., Newton, J.M. and Rowe, R.C. (2002) The evaluation of the rheological properties of lactose/microcrystalline cellulose and water mixtures by controlled stress rheometry and the relationship to the production of spherical pellets by extrusion-spheronisation, Eur. J. Pharm. Sci, 17, 43-50.

[21] Zhang, M., Rough, S.L., Ward, R., Seiler, C. and Wilson, D.I. (2011) A comparison of ram extrusion by single-holed and multi-holed dies for extrusion-spheronisation of microcrystalline-based pastes. Int. J. Pharm., 416, 210-222.

[22] Wilson, D.I. and Rough, S.L. (2012) Paste engineering: Multi-phase materials and multi-phase flows, Can. J. Chem. Eng., 90(2), 277-289.

[23] Kumar, G.V. and Muir Wood, D. (1999) Fall cone and compression tests on claygravel mixtures, Géotechnique, 49, 727-739.

[24] Chopra, R., Podczeck, F., Newton, J.M. and Alderborn, G. (2002) The influence of pellet shape and film coating on the filling of pellets into hard sphere capsules, Eur. $J$. Pharm. Biopharm., 53, 327-333.

[25] Chapman, S.R., Rowe, R.C. and Newton, J.M. (1988) Characterisation of the sphericity of particles by the one plane critical stability, J. Pharm. Pharmacol., 40(7), 503-506.

[26] Krueger, C., Thommes, M. and Kleinebudde, P. (2012) Spheronisation mechanism of MCC II-based pellets, Powder Tech., 238, 176-187. 


\section{Tables}

Table 1 Dimensionless groups for the spheronisation of extrudates

\begin{tabular}{|c|c|c|}
\hline Group & Attribute & Related to \\
\hline$\mu_{\mathrm{s},} \mu_{\mathrm{p}}$ & $\begin{array}{l}\text { Pellet-spheroniser surface and pellet- } \\
\text { pellet friction coefficients }\end{array}$ & $\begin{array}{l}\text { Frictional forces during pellet-wall } \\
\text { and pellet-pellet collisions }\end{array}$ \\
\hline$N$ & Number of pellets (initial value $=N_{0}$ ) & $\begin{array}{l}\text { Probability of collisions; mass of } \\
\text { pellets }\end{array}$ \\
\hline$\frac{\sigma_{\mathrm{Y}}}{\rho(\omega R)^{2}}$ & $\begin{array}{l}\text { Ratio of bulk yield strength to kinetic } \\
\text { energy per unit volume }\end{array}$ & $\begin{array}{l}\text { Deformation as a result of } \\
\text { dissipation of kinetic energy during } \\
\text { collisions }\end{array}$ \\
\hline$\frac{\omega R}{\sqrt{g D}}$ & Rotational Froude number & $\begin{array}{l}\text { Gravitational forces in pellet } \\
\text { motion }\end{array}$ \\
\hline$\frac{D}{R}$ & $\begin{array}{l}\text { Ratio of pellet minor axis }(b \approx D) \text { to } \\
\text { spheroniser friction plate radius }\end{array}$ & Pellet 'rope' thickness \\
\hline$\frac{D}{H}$ & $\begin{array}{l}\text { Ratio of pellet minor axis to spheroniser } \\
\text { friction plate element size }\end{array}$ & $\begin{array}{l}\text { Tendency for pellets to interact } \\
\text { with friction plate }\end{array}$ \\
\hline$\omega t$ & Angle travelled by friction plate & Cumulative work done \\
\hline
\end{tabular}


Table 2 Paste formulations and parameters

\begin{tabular}{lcccccccc}
\hline Label & $\begin{array}{c}\text { Symbol } \\
\text { in plots }\end{array}$ & $\begin{array}{c}\mathrm{MCC} \\
(\mathrm{wt} \%)\end{array}$ & $\begin{array}{c}\mathrm{CaCO}_{3} \\
(\mathrm{wt} \%)\end{array}$ & $\begin{array}{c}\text { Lactose } \\
(\mathrm{wt} \%)\end{array}$ & $\begin{array}{c}\text { Water } \\
(\mathrm{wt} \%)\end{array}$ & $\begin{array}{c}\text { Bulk } \\
\text { density }^{\mathrm{a}} \\
\left(\mathrm{kg} \mathrm{m}^{-3}\right)\end{array}$ & $\begin{array}{c}A R_{\text {end }} \\
-\end{array}$ & $\begin{array}{c}t_{\text {end }} \\
(\mathrm{s})\end{array}$ \\
\hline $\mathrm{M}^{\mathrm{b}}$ & $\diamond$ & 45 & 0 & 0 & 55 & 1170 & 0.95 & $130 \pm 10$ \\
$\mathrm{M}$ & 0 & 45 & 0 & 0 & 55 & 1155 & 0.96 & $45 \pm 15$ \\
MA5 & 0 & 43 & 5 & 0 & 52 & 1200 & 0.95 & $90 \pm 30$ \\
MA10 & 0 & 41 & 10 & 0 & 50 & 1240 & 0.93 & $150 \pm 30$ \\
MA15 & - & 38 & 15 & 0 & 47 & 1275 & 0.91 & $240 \pm 60$ \\
ML & $\triangle$ & 25 & 0 & 25 & 50 & 1310 & 0.96 & $110 \pm 10$ \\
\hline
\end{tabular}

${ }^{\mathrm{a}}$ calculated from compaction experiments, $\pm 10 \mathrm{~kg} \mathrm{~m}^{-3}$

${ }^{\mathrm{b}}$ data from Bryan et al. [1] 


\section{List of Figure Captions}

Figure 1 Bagley-type plots for the extrusion of paste MA15 ( $\left.D_{\mathrm{b}}=25 \mathrm{~mm}, D=3.5 \mathrm{~mm}\right)$. The size of the symbols is larger than the variation between repeated tests $( \pm 0.3 \mathrm{MPa})$. Best fit linear trendlines shown.

Figure 2 Effect of extrudate velocity on $(a)$ bulk yield strength and $(b)$ wall shear stress obtained from Benbow-Bridgwater characterisation of the pastes. Symbols are those in Table 2. Error bars in $(b)$ have been omitted for clarity (less than $\pm 0.02 \mathrm{MPa}$ ). Lines are drawn as a visual aid.

Figure 3 Effect of paste composition on bulk yield strength (circles, logarithmic scale) and density (triangles, linear scale). Large open symbols at $0 \mathrm{wt} \% \mathrm{CaCO}_{3}$ content indicate $\mathrm{MCC}$-lactose paste (ML). The size of the symbols is larger than the variation in parameters. Best fit trendlines shown.

Figure 4 Evolution of pellet aspect ratio during spheronisation for $(a)$ paste $\mathrm{M}$, taken from Bryan et al. [1], and (b) paste ML. Error bars show $10^{\text {th }}$ and $90^{\text {th }}$ percentile values of $A R$ distribution. Horizontal dashed lines indicate $A R_{\text {end }}$; vertical dashed lines indicate $t_{\text {end }}$. Solid locus on $(a)$ shows Model I, fitted to give $A R=0.95-0.35 \exp (-0.04 t)$; dotted locus shows Model II, fitted to give $A R=0.95-\left(0.35^{-1}+0.20 t\right)^{-1}$.

Figure 5 Evolution of pellet aspect ratio during spheronisation for MCC-carbonate pastes (MCClactose paste (ML) data shown in Figure 4(b)). Symbols are those in Table 2. Error bars show $10^{\text {th }}$ and $90^{\text {th }}$ percentile values of $A R$ distribution. Horizontal dashed lines indicate $A R_{\text {end }}$; vertical dashed lines indicate $t_{\text {end }}$.

Figure 6 Influence of $\sigma_{\mathrm{Y}} / \rho(\omega R)^{2}$ on the dimensionless spheronisation end parameters $(a) A R_{\text {end }}$ and $(b)$ $\omega t_{\text {end }}$. Horizontal error bars calculated using data in Figure 3. Vertical error bars in $(b)$ calculated using the ranges in $t_{\text {end }}$ reported in Table 2. Dashed lines show fit of the MCCcarbonate data to linear trends.

Figure 7 Evolution of pellet shape during spheronisation plotted in dimensionless form. Error bars show $10^{\text {th }}$ and $90^{\text {th }}$ percentile values of $A R / A R_{\text {end }}$ distribution. Dashed locus shows linear trends fitted to the data.

Figure 8 Radial (lower profile, $u_{\mathrm{r}}$ ) and azimuthal (upper profile, $u_{\theta}$ ) velocities of a paste $\mathrm{M}$ extrudate rod (see inset) tracked during the initial part of the breakage stage of a spheronisation test at $1200 \mathrm{rpm}$, using a time step, $\Delta t$, of $20 \mathrm{~ms}$. Label A indicates where the body was accelerated by the friction plate; ${ }^{*} \mathrm{w}$ and $*_{\mathrm{p}}$ indicate collision with the wall and a single pellet, respectively. The vertical dashed lines indicate the following stages: (1) acceleration of the rod by the friction plate, followed by rod tumbling; (2) breakage of the rod into two fragments (the tracking continues with the larger fragment); (3) acceleration of the larger fragment by the friction plate, followed by multiple collisions with other rods/fragments and the wall; and (4) the larger fragment moving with a cluster of other rods/fragments. The upper limit of the velocity scale, of $7.5 \mathrm{~m} \mathrm{~s}^{-1}$, is the rim speed of the spheroniser friction plate.

Figure 9 Radial (lower profile, $u_{\mathrm{r}}$ ) and azimuthal (upper profile, $u_{\theta}$ ) velocities of a paste M rod 
fragment (see inset) tracked during the rounding stage of a spheronisation test at $1200 \mathrm{rpm}$, using a time step, $\Delta t$, of $2 \mathrm{~ms}$. Time shown, $t^{\prime}$, is time elapsed from $t=20 \mathrm{~s}$. The vertical lines separate the following regimes: (1) the fragment experiences multiple accelerations from contact with the friction plate and collisions with other fragments/the wall; (2) the fragment moves as part of a cluster, above the friction plate; and (3) the fragment leaves the cluster and experiences motion similar to regime (1). The upper limit of the velocity scale, of $7.5 \mathrm{~m} \mathrm{~s}^{-1}$, is the rim speed of the spheroniser friction plate.

Figure S1 Data sets taken from Fielden et al. [18] plotted in similar form to Figure 4. Data sets showing the evolution of the One Plane Critical Stability (OPCS) measure of roundness [25] (where a sphere has OPCS =1) are plotted as $\mathrm{OPCS}^{-1}$ against time. Labels 'fine' and 'coarse' refer to the lactose powder particle size distribution.

Figure S2 Data set for spheronisation of MCC-I (20\%) and lactose (80\%) material extracted from Krueger et al. [26] plotted in similar form to Figure 4. Spheronisation tip speed $14 \mathrm{~m} \mathrm{~s}^{-1}$. Data were reported in the form of $A R^{-1}$.

Figure S3 Data sets from Koester et al. [8] plotted in similar form to Figure 4. Data were reported in the form of $A R^{-1}$. 


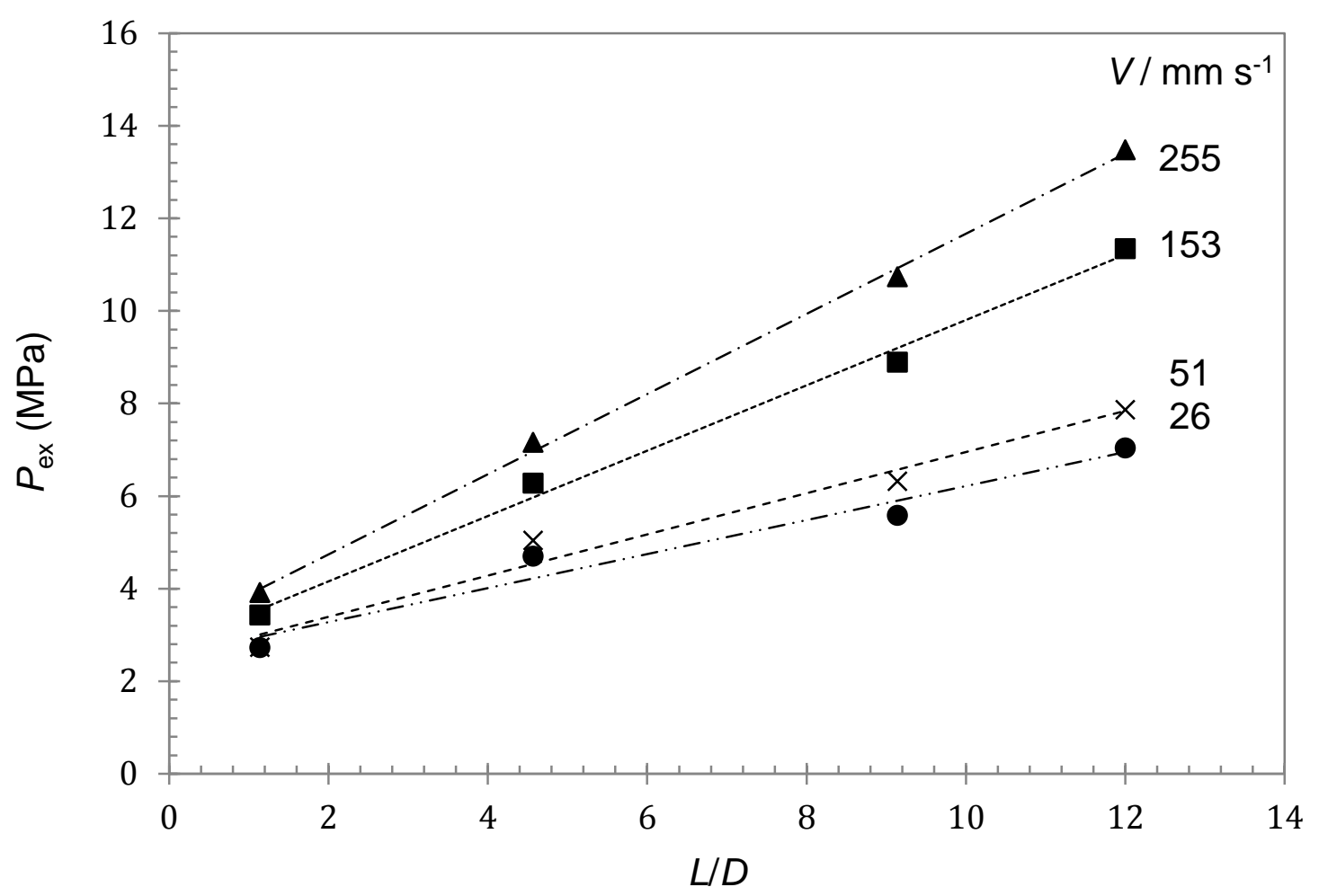

Figure 1 Bagley-type plots for the extrusion of paste MC15 $\left(D_{\mathrm{b}}=25 \mathrm{~mm}, D=3.5 \mathrm{~mm}\right)$. The size of the symbols is larger than the variation between repeated tests $( \pm 0.3 \mathrm{MPa})$. Best fit linear trendlines shown. 
(a)

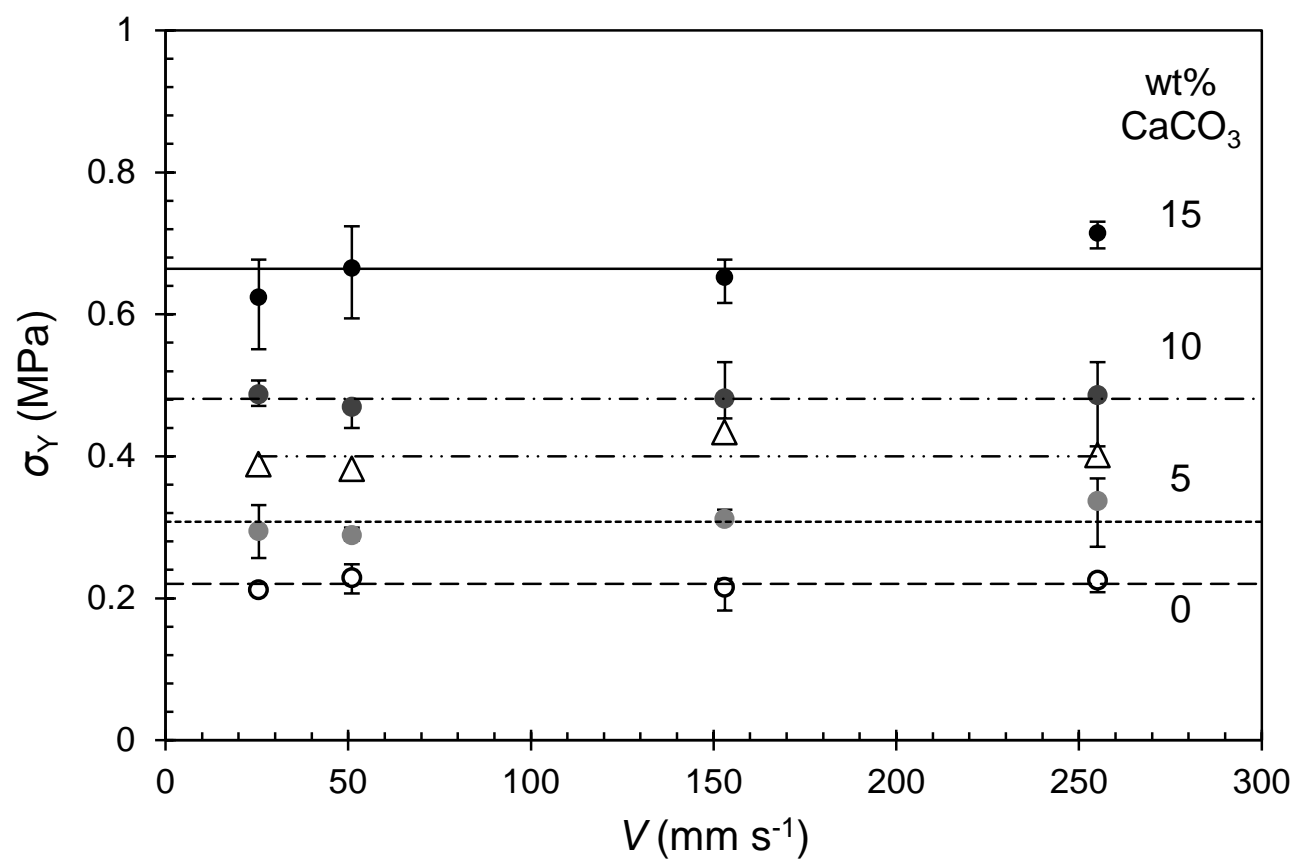

(b)

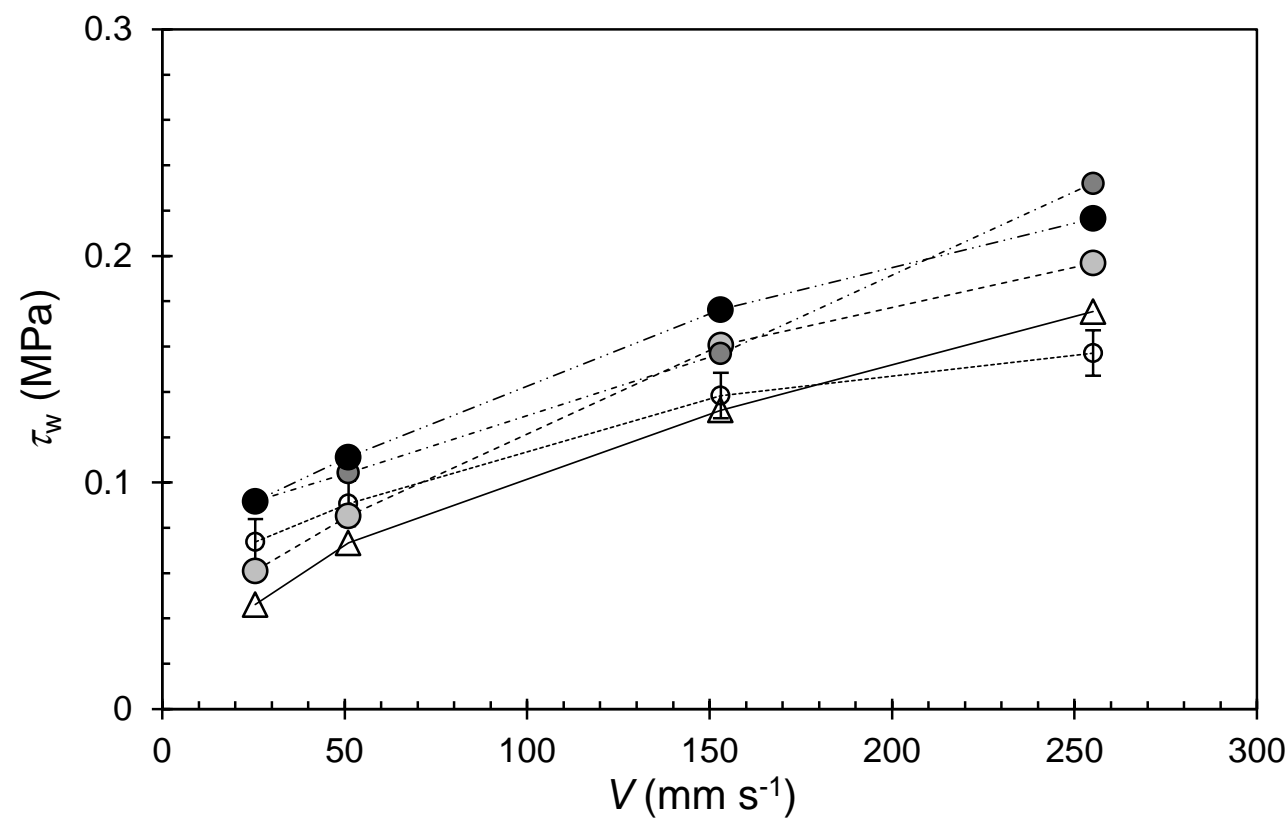

Figure 2 Effect of extrudate velocity on $(a)$ bulk yield strength and $(b)$ wall shear stress obtained from Benbow-Bridgwater characterisation of the pastes. Symbols are those in Table 2. Error bars in $(b)$ have been omitted for clarity (less than $\pm 0.02 \mathrm{MPa}$ ). Lines are drawn as a visual aid. 


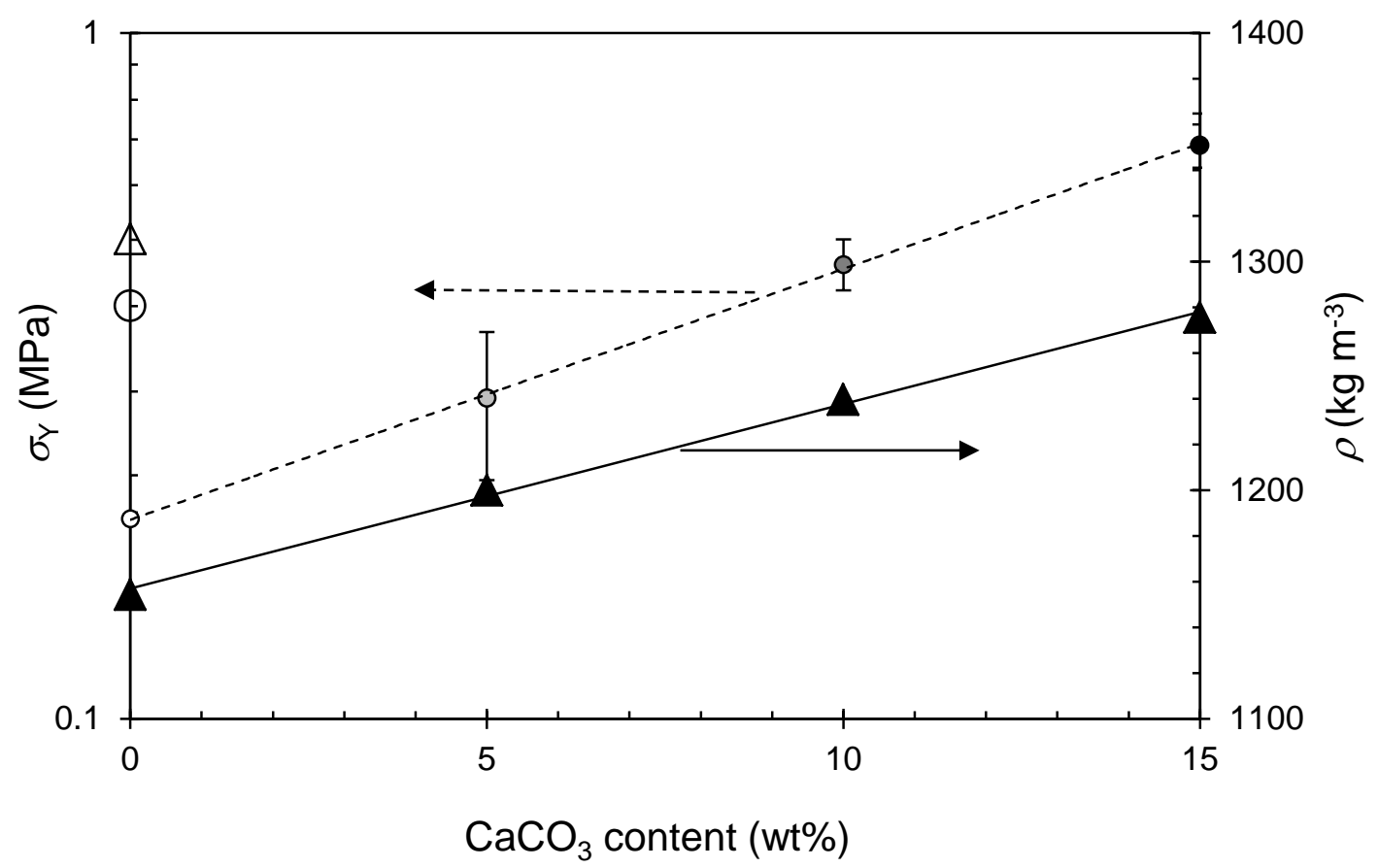

Figure 3 Effect of paste composition on bulk yield strength (circles, logarithmic scale) and density (triangles, linear scale). Large open symbols at $0 \mathrm{wt} \% \mathrm{CaCO}_{3}$ content indicate $\mathrm{MCC}$-lactose paste (ML). The size of the symbols is larger than the variation in parameters. Best fit trendlines shown. 
(a)

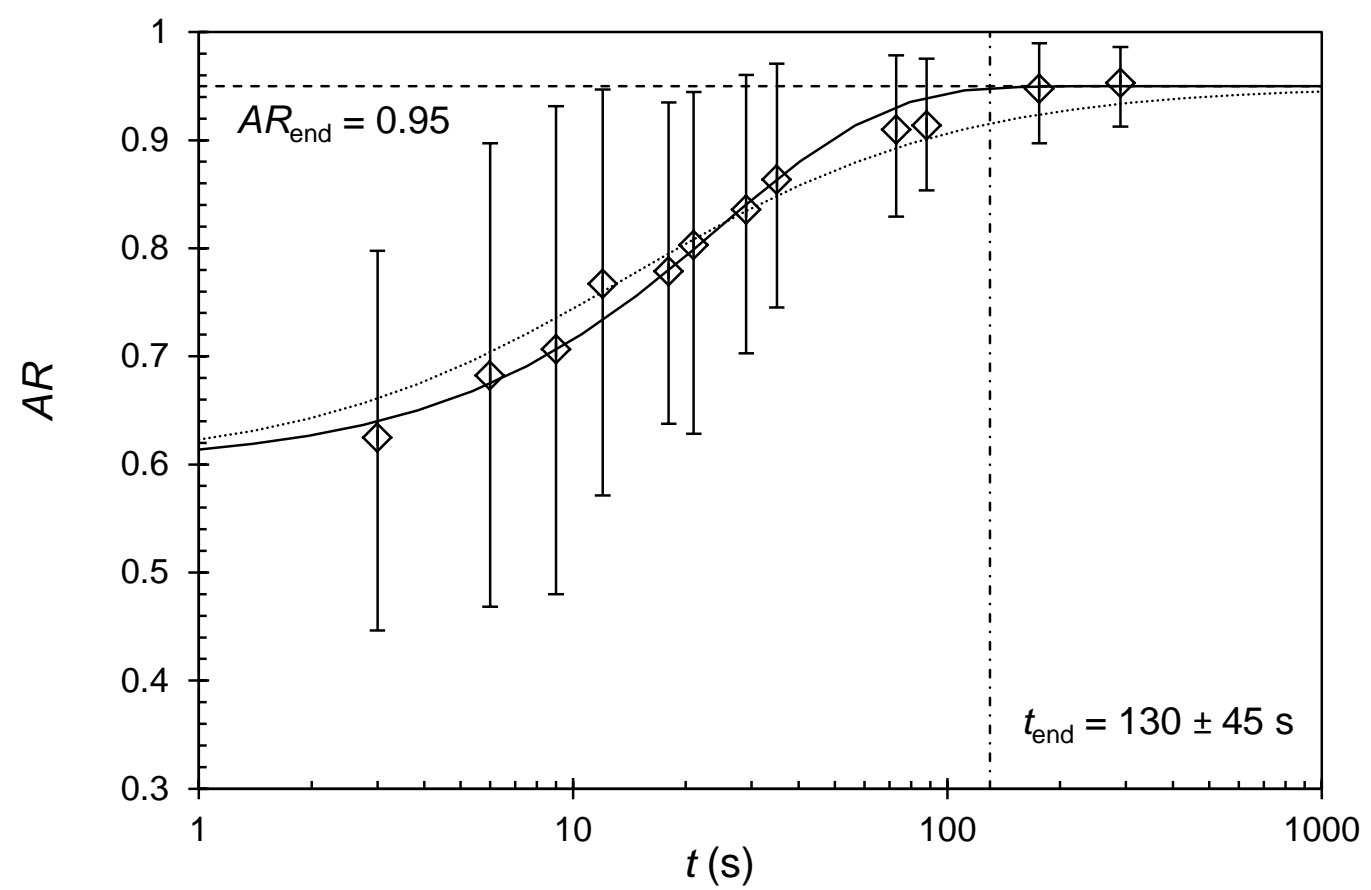

(b)

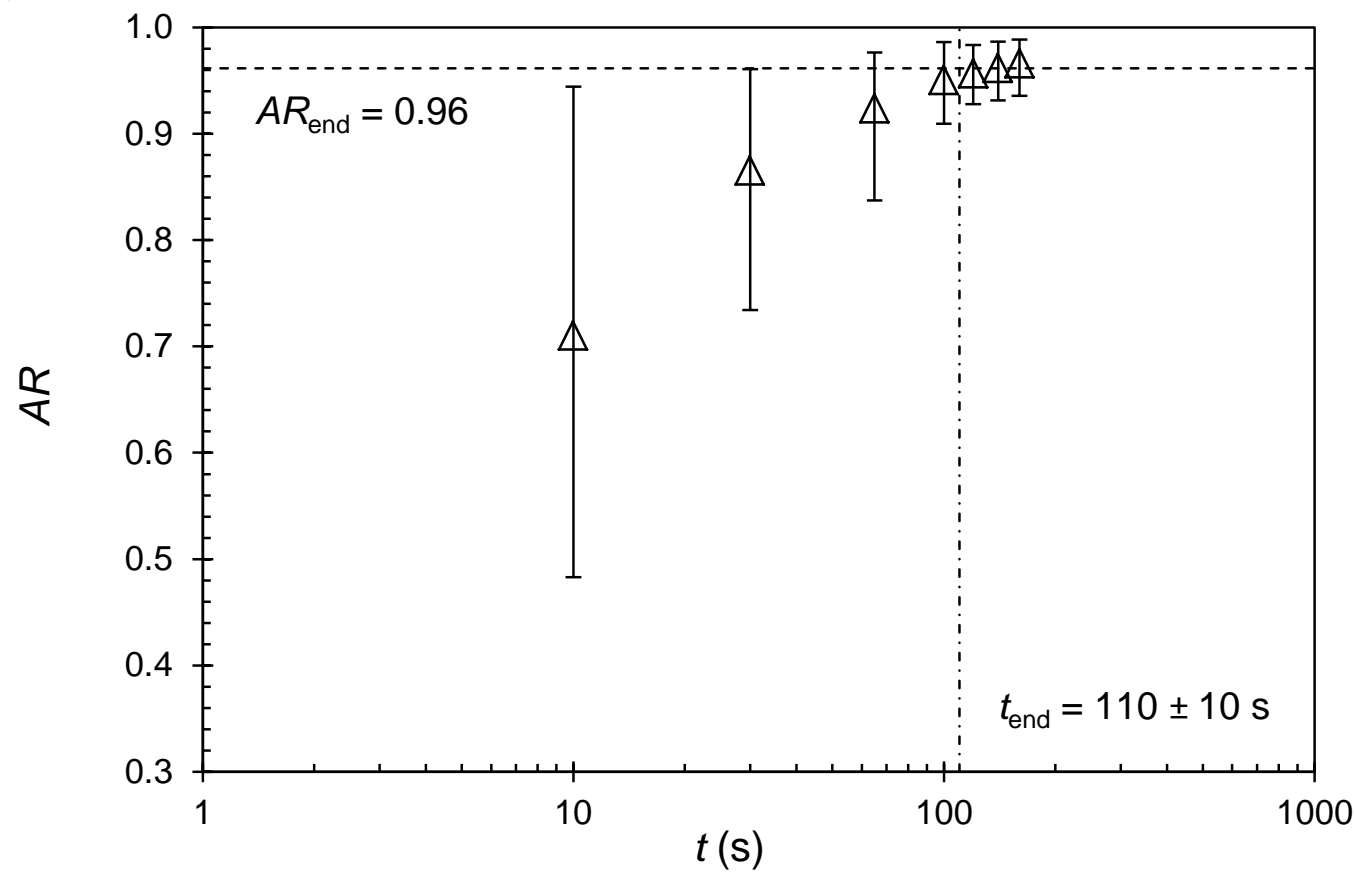

Figure 4 Evolution of pellet aspect ratio during spheronisation for (a) paste M, taken from Bryan et al. (2015a), and $(b)$ paste ML. Error bars show $10^{\text {th }}$ and $90^{\text {th }}$ percentile values of $A R$ distribution. Horizontal dashed lines indicate $A R_{\text {end }}$; vertical dashed lines indicate $t_{\text {end }}$. Solid locus on $(a)$ shows Model I, fitted to give $A R=0.95-0.35 \exp (-0.04 t)$; dotted locus shows Model II, fitted to give $A R=0.95-\left(0.35^{-1}+0.20 t\right)^{-1}$. 
(a) $\mathrm{M}$

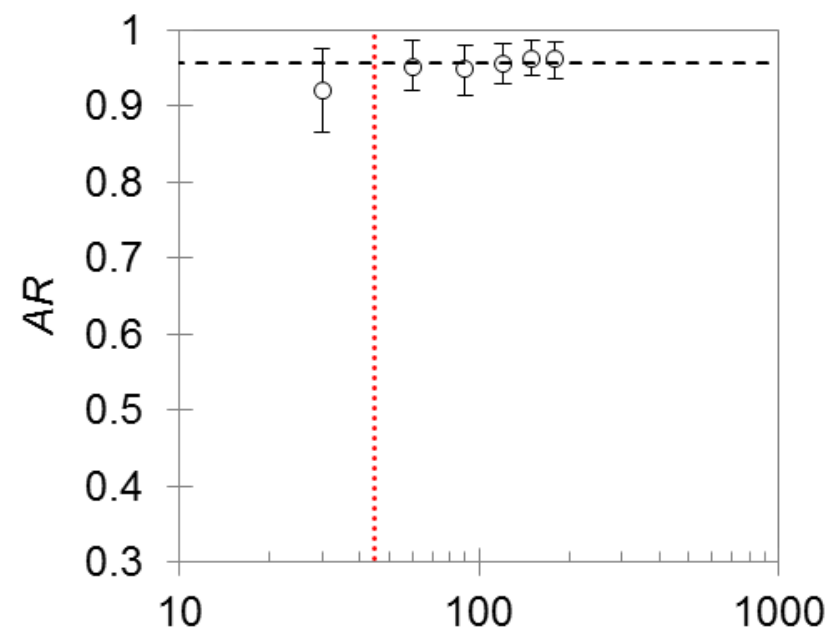

(c) MA10

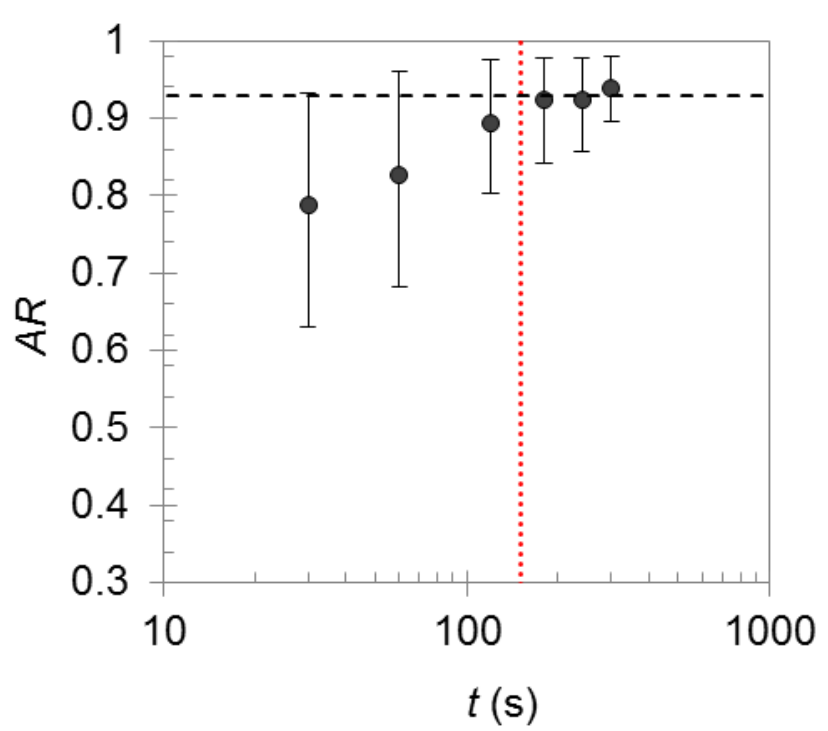

(b) MA5

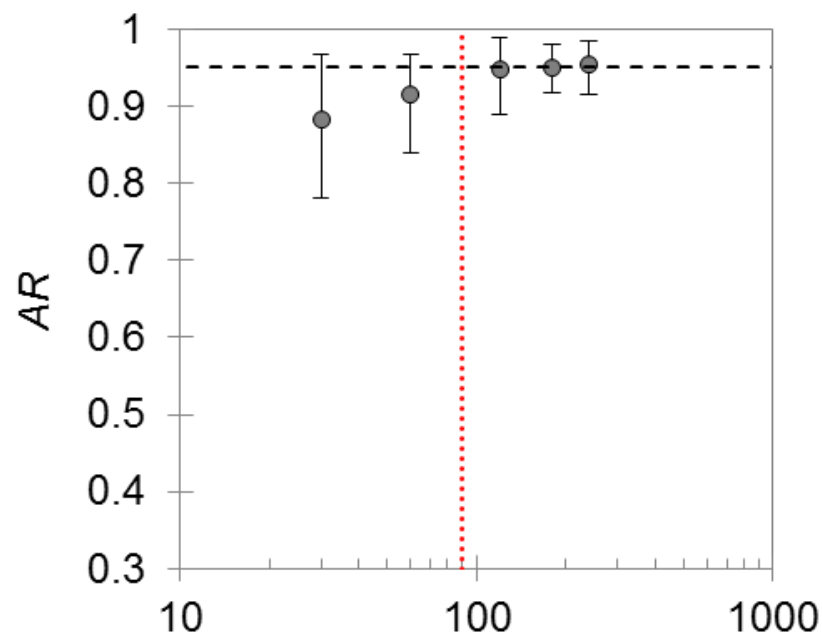

(d) MA15

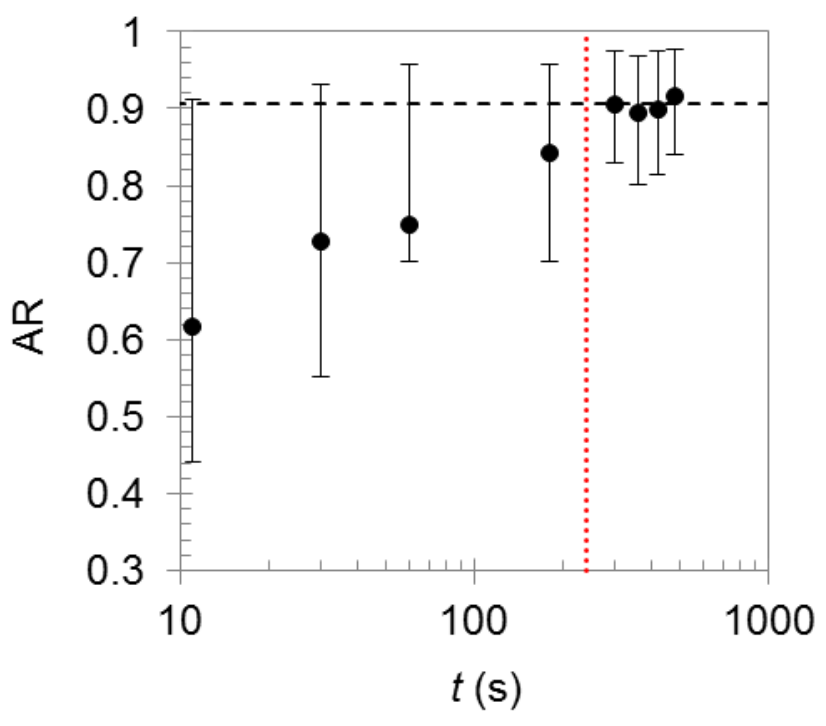

Figure 5 Evolution of pellet aspect ratio during spheronisation for MCC-carbonate pastes (MCClactose paste (ML) data shown in Figure 4(b)). Symbols are those in Table 2. Error bars show $10^{\text {th }}$ and $90^{\text {th }}$ percentile values of $A R$ distribution. Horizontal dashed lines indicate $A R_{\text {end }}$; vertical dashed lines indicate $t_{\text {end }}$. 
(a)

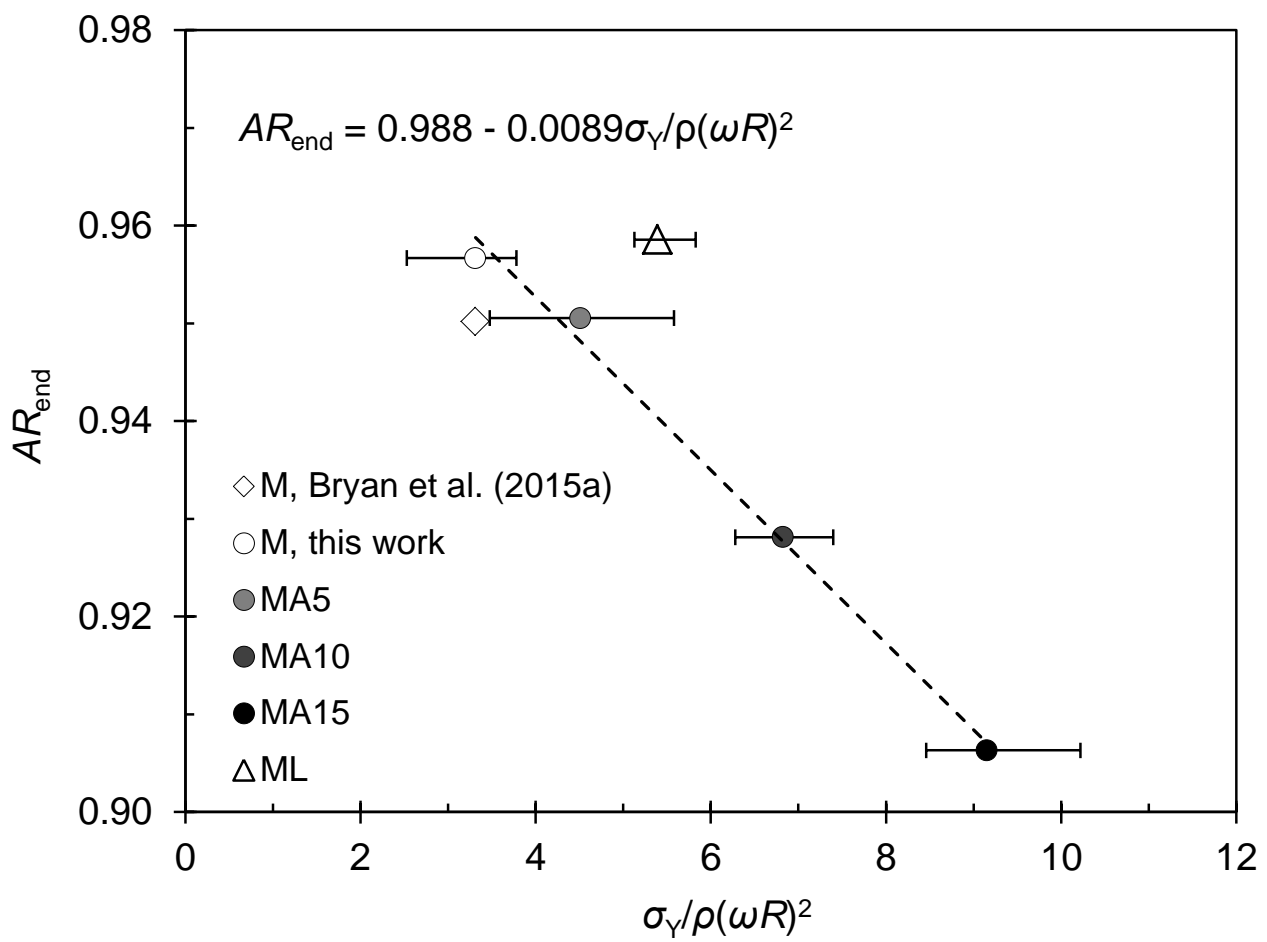

(b)

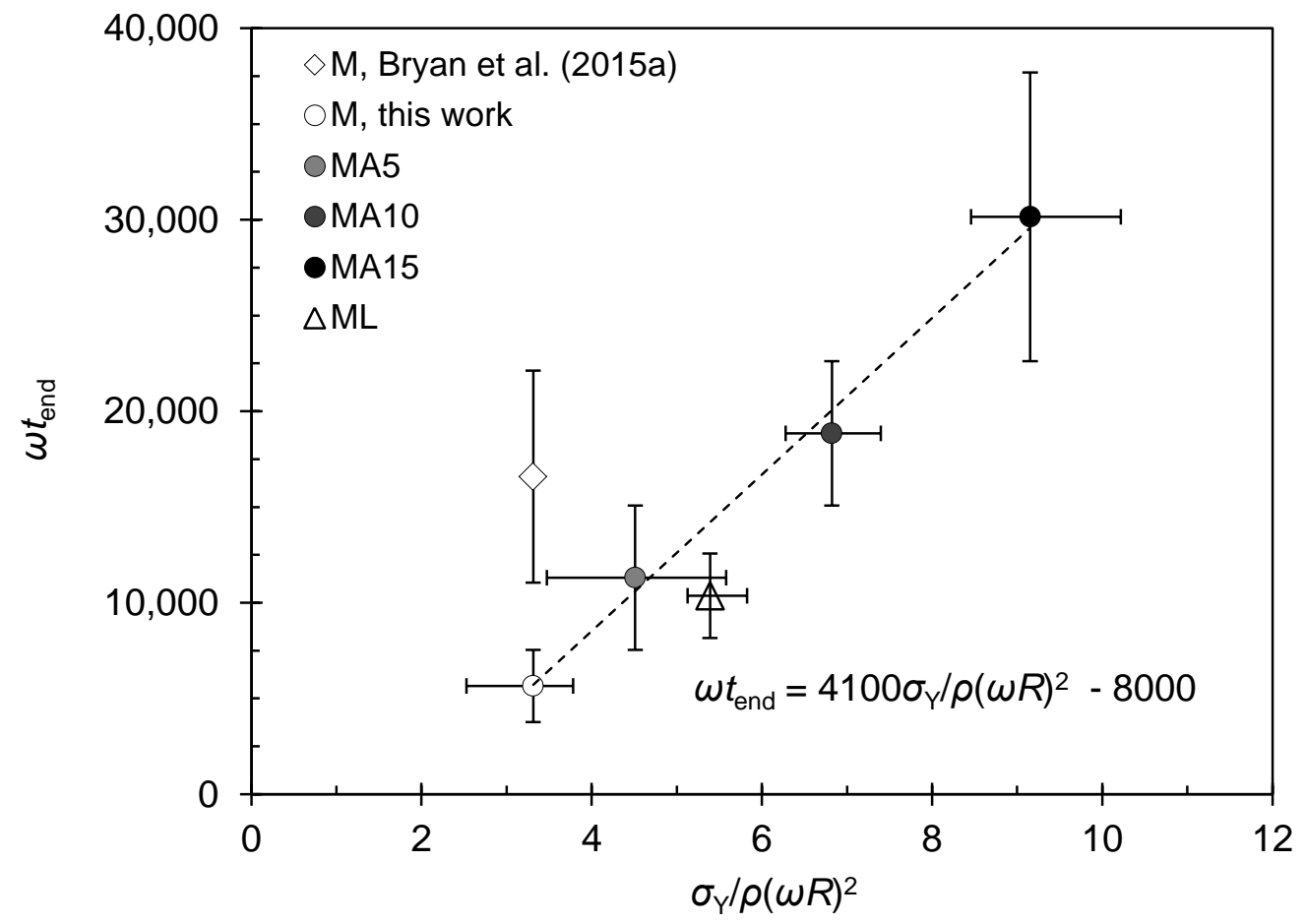

Figure 6 Influence of $\sigma_{\mathrm{Y}} / \rho(\omega R)^{2}$ on the dimensionless spheronisation end parameters $(a) A R_{\text {end }}$ and $(b)$ $\omega t_{\text {end }}$. Horizontal error bars calculated using data in Figure 3. Vertical error bars in $(b)$ calculated using the ranges in $t_{\text {end }}$ reported in Table 2. Dashed lines show fit of the MCCcarbonate data to linear trends. 


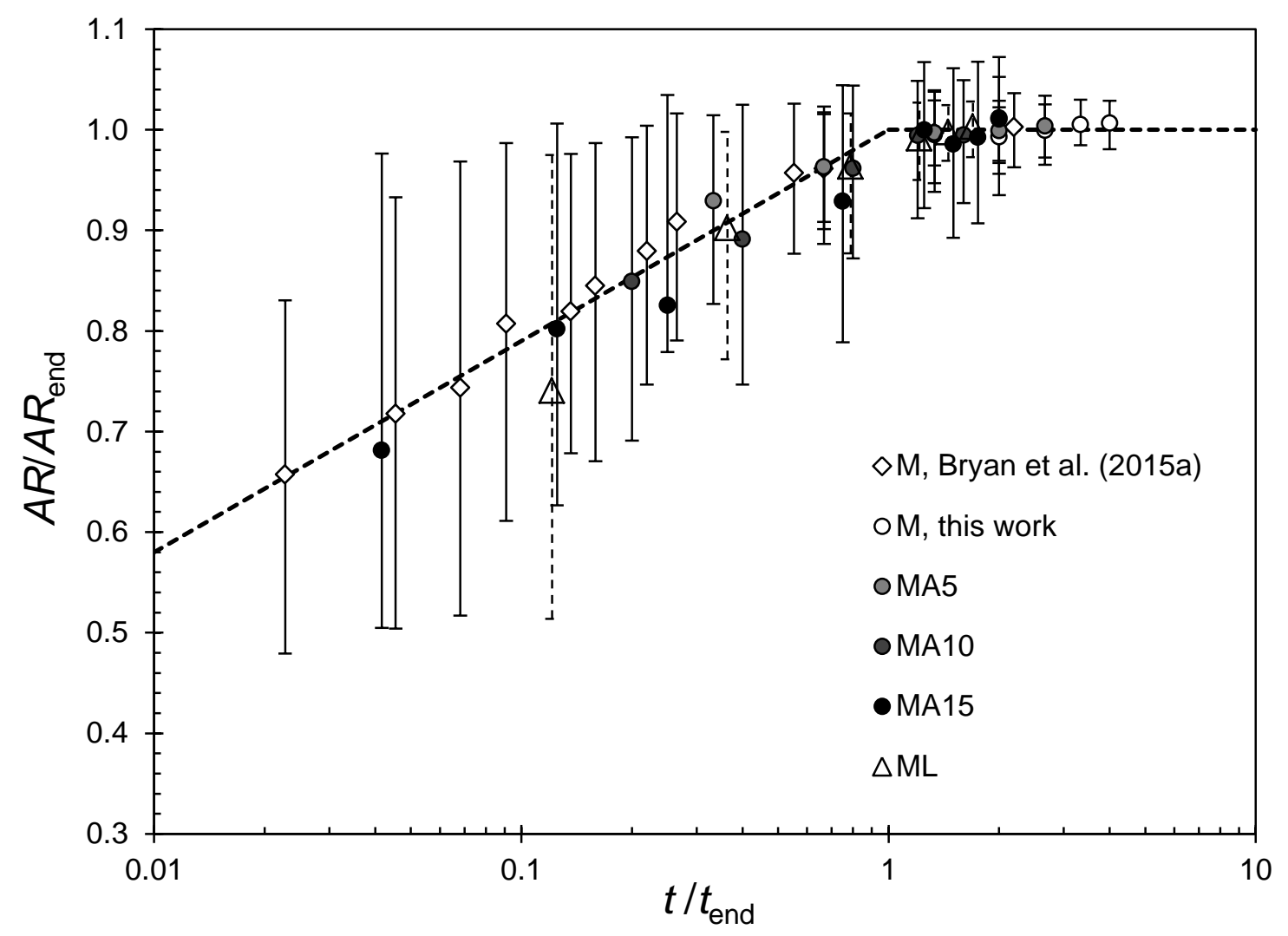

Figure 7 Evolution of pellet shape during spheronisation plotted in dimensionless form. Error bars show $10^{\text {th }}$ and $90^{\text {th }}$ percentile values of $A R / A R_{\text {end }}$ distribution. Dashed locus shows linear trends fitted to the data. 


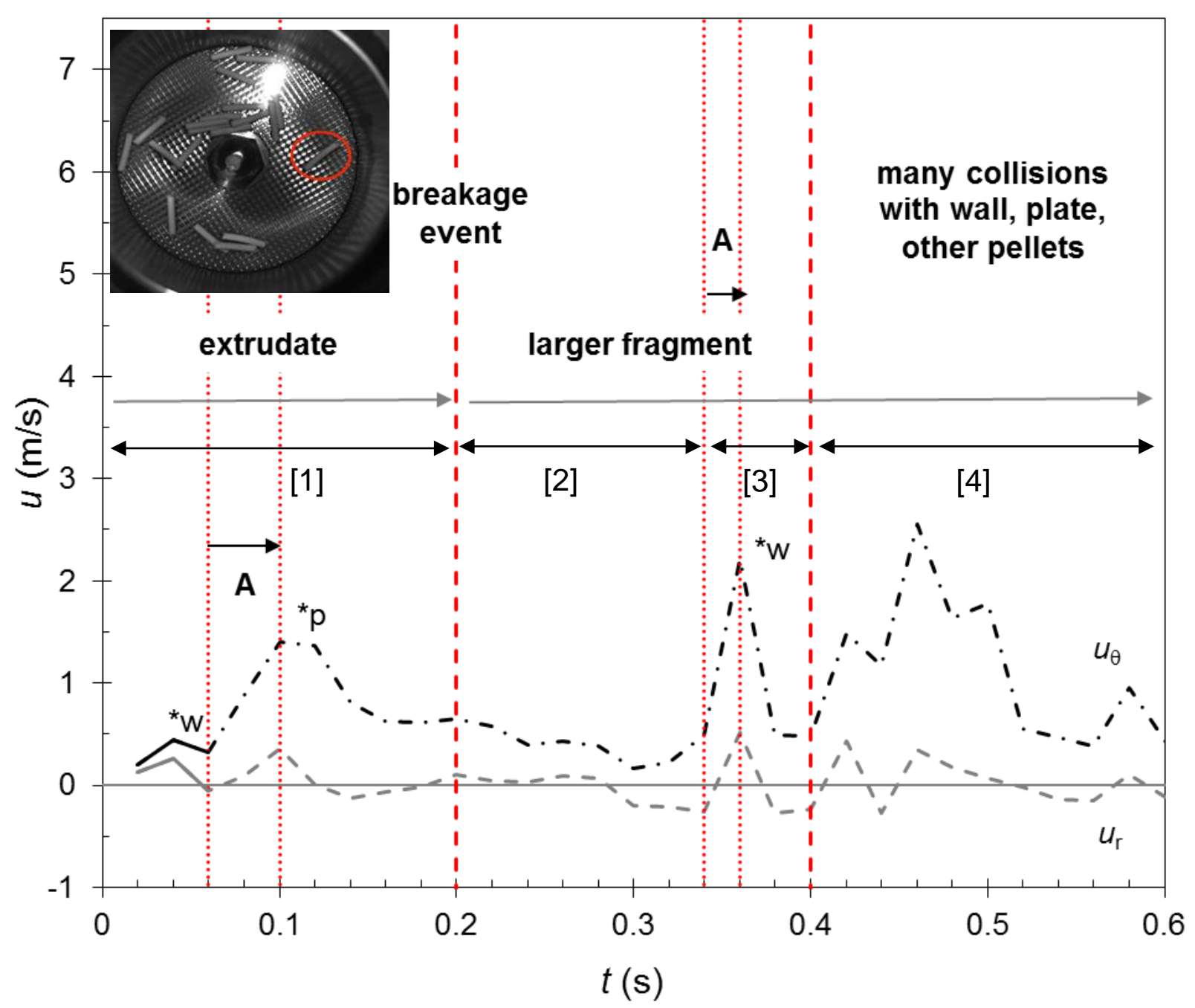

Figure 8 Radial (lower profile, $u_{\mathrm{r}}$ ) and azimuthal (upper profile, $u_{\theta}$ ) velocities of a paste $\mathrm{M}$ extrudate rod (see inset) tracked during the initial part of the breakage stage of a spheronisation test at $1200 \mathrm{rpm}$, using a time step, $\Delta t$, of $20 \mathrm{~ms}$. Label A indicates where the body was accelerated by the friction plate; ${ }^{*} \mathrm{w}$ and $*_{\mathrm{p}}$ indicate collision with the wall and a single pellet, respectively. The vertical dashed lines indicate the following stages: [1] acceleration of the rod by the friction plate, followed by rod tumbling; [2] breakage of the rod into two fragments (the tracking continues with the larger fragment); [3] acceleration of the larger fragment by the friction plate, followed by multiple collisions with other rods/fragments and the wall; and [4] the larger fragment moving with a cluster of other rods/fragments. The upper limit of the velocity scale, of $7.5 \mathrm{~m} \mathrm{~s}^{-1}$, is the rim speed of the spheroniser friction plate. 


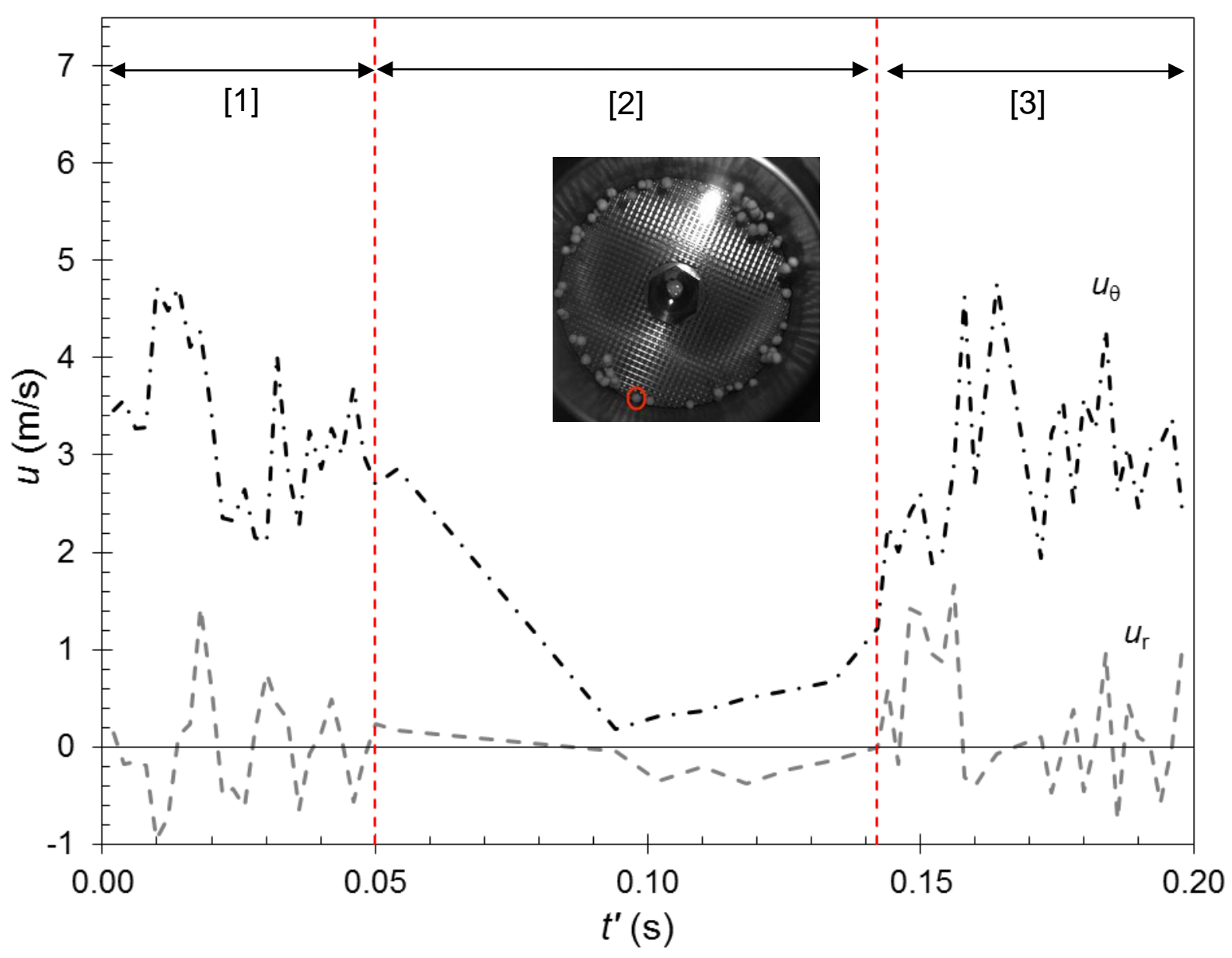

Figure 9 Radial (lower profile, $u_{\mathrm{r}}$ ) and azimuthal (upper profile, $u_{\theta}$ ) velocities of a paste $\mathrm{M}$ rod fragment (see inset) tracked during the rounding stage of a spheronisation test at $1200 \mathrm{rpm}$, using a time step, $\Delta t$, of $2 \mathrm{~ms}$. Time shown, $t^{\prime}$, is time elapsed from $t=20 \mathrm{~s}$. The vertical lines separate the following regimes: [1] the fragment experiences multiple accelerations from contact with the friction plate and collisions with other fragments/the wall; [2] the fragment moves as part of a cluster, above the friction plate; and [3] the fragment leaves the cluster and experiences motion similar to regime [1]. The upper limit of the velocity scale, of $7.5 \mathrm{~m} \mathrm{~s}^{-1}$, is the rim speed of the spheroniser friction plate. 


\section{Supplementary Material}

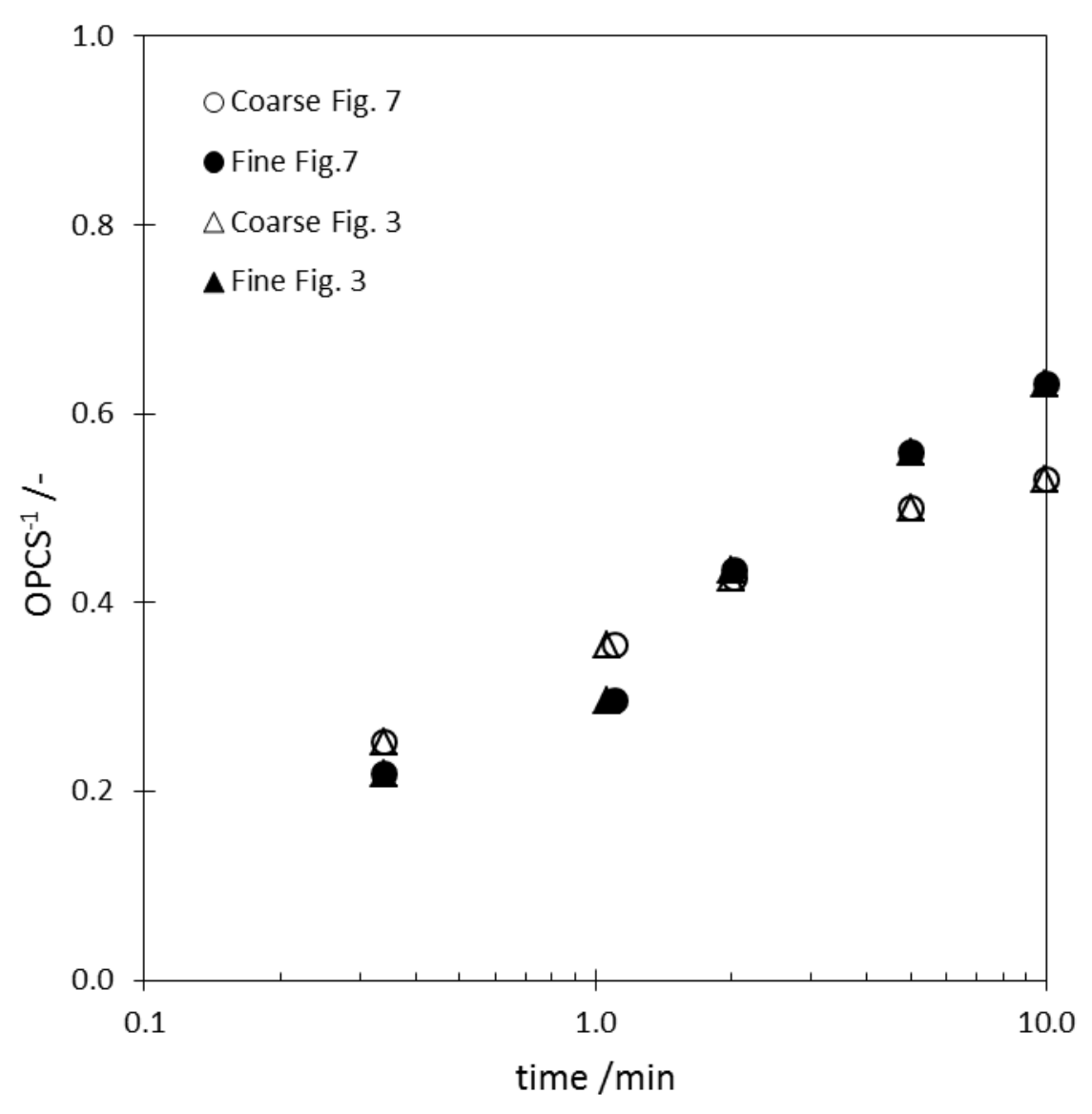

Figure S1. Data sets taken from Fielden et al. (1992) plotted in similar form to Figure 4. Data sets showing the evolution of the One Plane Critical Stability $\left(\mathrm{OPCS}^{1}\right)$ measure of roundness (where a sphere has OPCS $=1$ ) are plotted as $\mathrm{OPCS}^{-1}$ against time. Labels 'fine' and 'coarse' refer to the lactose powder particle size distribution.

Fielden, K.E., Newton, J.M. and Rowe, R.C. (1992) The influence of lactose particle size on spheronization of extrudate processed by a ram extruder, Int. J. Pharm., 81, 205-224.

\footnotetext{
${ }^{1}$ Chapman, S.R., Rowe, R.C. and Newton, J.M. (1988) Characterisation of the sphericity of particles by the one plane critical stability, J. Pharm. Pharmacol., 40(7), 503-506.
} 


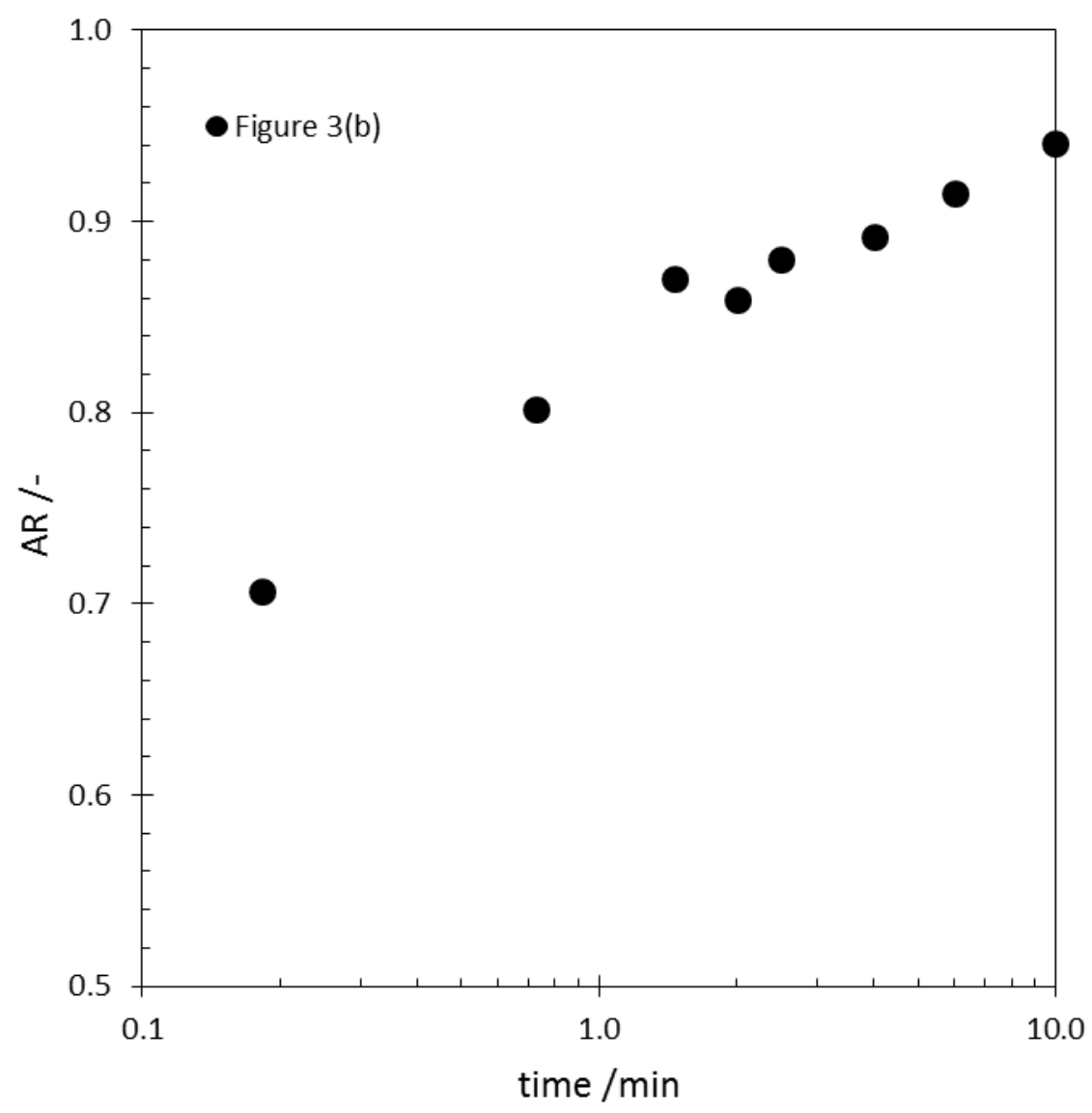

Figure S2. Data set for spheronisation of MCC-I (20\%) and lactose (80\%) material extracted from Krueger et al. (2012) plotted in similar form to Figure 4. Spheronisation tip speed $14 \mathrm{~m} \mathrm{~s}^{-1}$. Data were reported in the form of $A R^{-1}$.

Krueger, C., Thommes, M. and Kleinebudde, P. (2012) Spheronisation mechanism of MCC IIbased pellets, Powder Tech., 238, 176-187. 


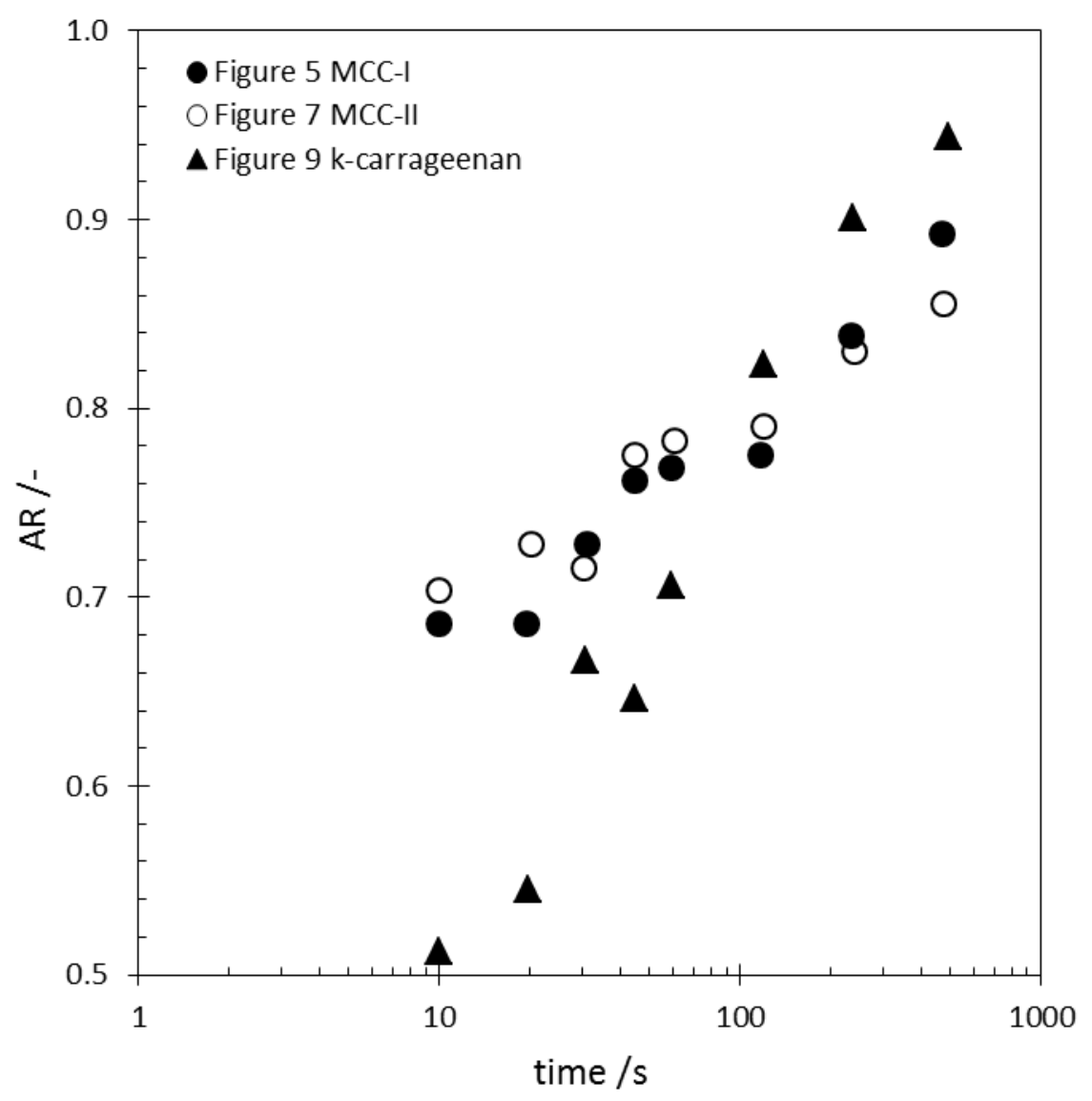

Figure S3. Data sets from Koester et al. (2012) plotted in similar form to Figure 4. Data were reported in the form of $A R^{-1}$.

Koester, M., Willemsen, E., Krueger, C. and Thommes, M. (2012) Systematic evaluations regarding interparticle mass transfer in spheronization, Int. J. Pharm., 431, 84-89. 